\title{
The Impact of Multiperiod Planning Horizons on Portfolios and Asset Prices in a Dynamic CAPM*
}

\author{
Marten Hillebrand \\ Jan Wenzelburger \\ Bielefeld Graduate School of \\ Fakultät für \\ Economics and Management \\ Wirtschaftswissenschaften
Universität Bielefeld, Postfach 100131
D-33501 Bielefeld, Germany
marten.hillebrand@uni-bielefeld.de
jwenzelb@wiwi.uni-bielefeld.de

Discussion Paper No. 520

\begin{abstract}
This paper investigates a financial market in which investors with linear meanvariance preferences and multiperiod planning horizons of arbitrary finite length interact. Given heterogeneous subjective beliefs, the temporary equilibrium map determining market clearing prices is calculated explicitly. The classical capital market line result of CAPM theory is extended showing that under homogeneous beliefs investors with identical multiperiod planning horizons hold portfolios with equal proportions of risky assets. The existence of perfect forecasting rules for first and second moment beliefs which generate rational expectations is established.
\end{abstract}

Keywords: CAPM, financial markets, multiperiod portfolio decisions, rational expectations.

JEL Classification: E17, G12, O16

First version: Jul. 2004, this version: Jan. 2005.

\footnotetext{
*Acknowledgment. We are indebted to Volker Böhm, Jochen Jungeilges, and Thorsten Pampel for many discussions and valuable comments.
} 


\section{Introduction}

A typical feature of financial markets is that traders will have different planning horizons when investing their wealth in assets. It is intuitively clear that the length of the planning horizon will affect investors' risk taking behavior and thus their portfolio decisions. Investors with long planning horizons are likely to invest more wealth into risky assets than those with short horizons. In particular, institutional investors will pursue longterm strategies rather than trying to follow a momentary trend. An open issue for agent-based models with incomplete markets is to understand the impact of different planning horizons on the dynamics of asset prices, asset returns, and portfolio holdings and the impact of investors who are allowed to revise their portfolio plans over time to incorporate the arrival of new and unexpected information. The analysis of these effects requires a tractable model in which investors have heterogeneous multiperiod planning horizons.

Starting with the work of Markowitz (1952) and Tobin (1958), economists have investigated portfolio decisions which, given a certain expected return, minimize the risk of future wealth fluctuations. Based on this portfolio theory, Sharpe (1964), Lintner (1965) and Mossin (1966) developed the famous Capital Asset Pricing Model (CAPM). The CAPM has been extended by Stapleton \& Subrahmanyam (1978) to the case in which investors face a multiperiod rather than a single-period planning horizon. These models, however, remain inherently static and the results depend significantly on the assumption that beliefs of all investors are homogeneous and rational. All investors face the same multiperiod planning horizon. These assumptions preclude an analysis of how distinct planning horizons with possibly heterogeneous beliefs affect individual portfolio decisions and how the trading behavior of investors with different planning horizons affects asset prices. Most studies of multiperiod portfolio decisions in the literature as, for example, Chen, Jen \& Zionts (1971), Hakansson (1970, 1983), Ingersoll (1987) or Pliska (1997) consider an essentially static one-shot optimization. The possibility to re-optimize previously made portfolio plans is ruled out. Scenarios in which investors permanently update subjective beliefs and for this reason want to revise their portfolios are not considered.

The present paper addresses these issues and is based on work of Böhm, Deutscher \& Wenzelburger (2000), Böhm \& Chiarella (2000), Wenzelburger (2004), and Hillebrand (2003). The key feature is that asset prices are endogenously determined by the demand behavior of traders. This allows for a fully explicit dynamic analysis of a financial market where investors may be arbitrarily heterogeneous with respect to their individual beliefs as well as their usual microeconomic characteristics like preferences and endowments. The trading behavior of all agents is described by asset demand functions which are derived from individual optimization problems. From these demand functions an explicit temporary equilibrium map is derived determining market clearing prices in each period. By employing the concept of a forecasting rule, the expectations formation of all investors is made explicit. Combined with these forecasting rules, an explicit time-one map of a stochastic difference equation is obtained in which expectations feed back into the actual 
evolution of asset prices, portfolios, and expectations.

In this spirit our analysis proceeds in three steps: First, we consider the individual portfolio choice problem of a single investor with a planning horizon of arbitrary finite length given subjective beliefs. Assuming linear mean-variance preferences, we compute an explicit asset demand function depending on beliefs. This provides a first insight how planning horizons of different length affect the demand behavior of investors. In a second step, a temporary equilibrium map determining market-clearing prices is derived from the aggregate excess demand function. The classical capital market line result of CAPM theory is extended to the case with multiperiod planning horizons by showing that portfolios of investors with homogeneous beliefs and identical planning horizons contain equal proportions of risky assets while different planning horizons will generally lead to structurally different portfolios. In a third step, the individual demand functions and the price law are embedded into a sequential model, taking proper account of how the individual demand behavior changes with new information and observations. By allowing for re-optimization of portfolio decisions, we thus obtain a dynamic description of how prices and portfolios evolve over time. Introducing forecasting rules that provide correct first and second moments of the evolving price process, we relate our setup to the more traditional CAPM. These forecasting rules generate rational expectations for a group of investors in the sense that the subjective first two moments coincide with the true first two moments while the beliefs of other market participants may be false.

The remainder of this paper is organized as follows. Section 2 is concerned with the multiperiod portfolio choice problem of investors with linear mean-variance preferences. An explicit temporary equilibrium map describing market-clearing prices is computed. Section 3 treats the case with homogeneous expectations. Section 4 is concerned with the existence of forecasting rules which generate rational expectations, the dynamics of prices and portfolios under rational expectations are studied in Section 5. Conclusions are found in Section 6, the mathematical proof of the main theorem is placed in the appendix. 


\section{The Model}

\subsection{Overlapping cohorts of investors}

Consider a financial market in which a population of overlapping cohorts of investors who trade in discrete time periods. The set of investors in each period is composed of $J+1$ different cohorts or generations. ${ }^{1}$ In each trading period $t \in \mathbb{N}$, a new young cohort enters the market and trades for $J+1$ consecutive periods before its members exit the market to consume terminal wealth in period $t+J$. Each cohort will be identified by the index $j=0,1, \ldots, J$ describing the number of periods they remain in the market until their members exit. In particular, $j=J$ refers to the young and $j=0$ to the old cohort. Each cohort $j$ consists of $I$ types of investors characterized by risk preferences and subjective beliefs regarding the future evolution of the market. More precisely, a single investor in an arbitrary period is identified by the pair $(i, j)$ describing his type $i \in\{1, \ldots, I\}$ and his cohort $j \in\{0,1, \ldots, J\}$. Excluding the old cohort $j=0$, the set of investors trading in the market in each period is given by $\mathbb{I}:=\{1, \ldots, I\} \times\{1, \ldots, J\}$. The population structure in an arbitrary trading period $t \in \mathbb{N}$ is depicted in Figure 1.

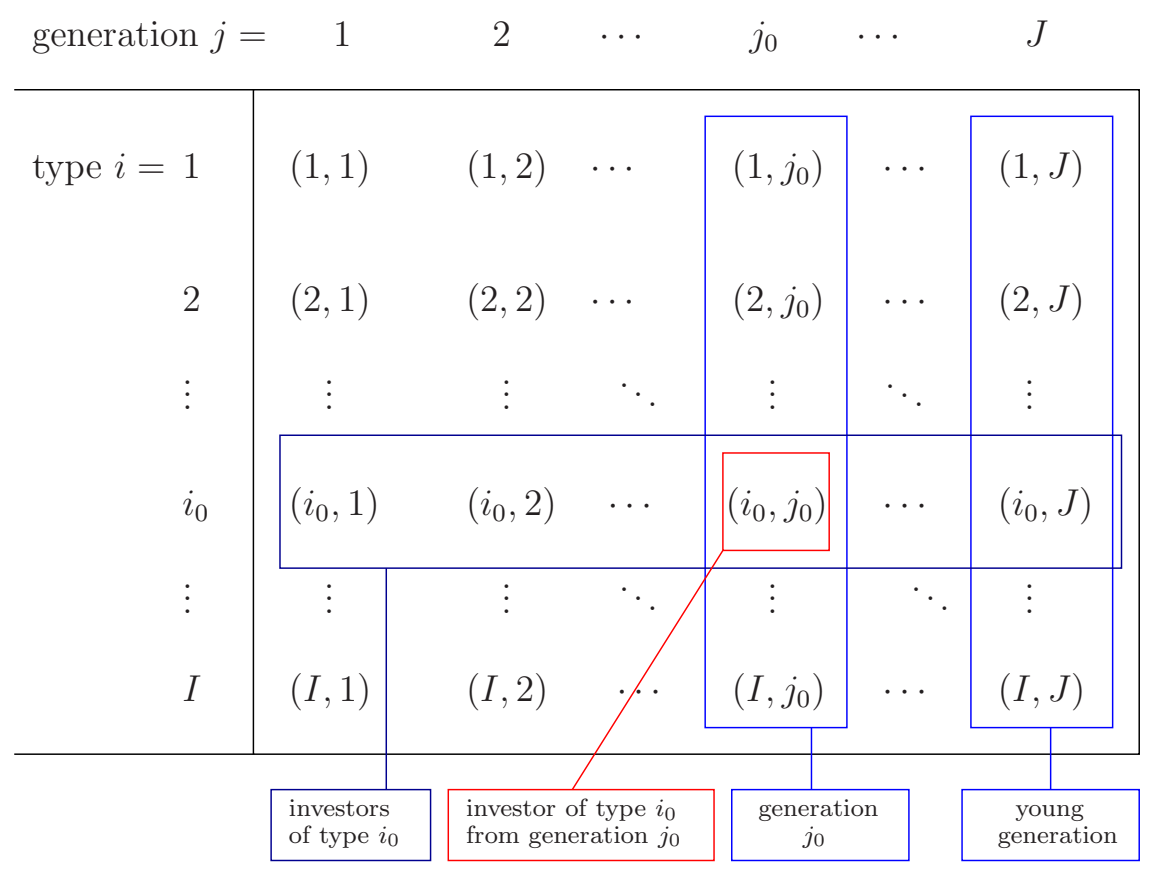

Figure 1: The investors in an arbitrary trading period $t$.

There is a single consumption good in the economy which serves as numeraire for all prices and payments. At the beginning of each period, any young investor $(i, J) \in \mathbb{I}$ of type $i$ receives an initial endowment of $e^{(i)}>0$ units of the consumption good. These endowments may depend on the type $i$ but are constant over time. Investors $(i, j)$ with $j<J$ do not receive endowments. Assuming that the consumption good cannot be

\footnotetext{
${ }^{1}$ One may think of a multiperiod OLG model, but in the context of this model this is not necessary.
} 
stored by consumers directly, each investor faces the problem of transferring wealth from the first to the last period of life in which he consumes the proceeds of his investments. There exist $K+1$ retradeable assets in the economy, indexed by $k=0,1, \ldots, K$. The first asset $k=0$ is a risk-less bond which pays a constant return $R>0$ per unit invested in the previous period. The assets $k=1, \ldots, K$ correspond to risky shares of firms which are traded at prices $p_{t}=\left(p_{t}^{(1)}, \ldots, p_{t}^{(K)}\right)^{\top} \in \mathbb{R}^{K}$ of period $t$. For simplicity, we abstract from dividend payments. In each trading period $t$, another group of so-called noise-traders who purchase or sell a random quantity $\xi_{t} \in \mathbb{R}^{K}$ is present in the market. ${ }^{2}$

\section{Assumption 1}

The portfolios of noise traders are given by an $\mathbb{R}^{K}$-valued stationary ergodic stochastic process $\left\{\xi_{t}\right\}_{t \in \mathbb{N}}$ on a the probability space $(\Omega, \mathcal{F}, \mathbb{P})$, which is adapted to the filtration $\left\{\mathcal{F}_{t}\right\}_{t \in \mathbb{N}}$ such that each $\xi_{t}$ is $\mathcal{F}_{t}$ measurable.

\subsection{Decision problem}

Consider first the portfolio choice problem faced by an investor $(i, j) \in \mathbb{I}$ in an arbitrary period $t$ with planning horizon $t+j$. At the beginning of period $t$ any investor forms beliefs regarding future prices $p_{t+1}, \ldots, p_{t+j}$ which are relevant for her portfolio choices. These beliefs are given by a subjective joint probability distribution for the random variables $p_{t+1}, \ldots, p_{t+j}$. Given her beliefs, the investor's portfolio decision will depend on current prices as well on her wealth position in period $t$. We assume that the portfolio problem in period $t$ is solved prior to trading, that is, before the actual price $p_{t}$ has been observed and before the noise traders' transaction $\xi_{t}$ has been realized. Current prices will therefore enter the decision problem as a parameter $p \in \mathbb{R}^{K}$. To determine the investors initial wealth position at time $t$ we need to distinguish between young and non-young investors. Each young investor's wealth is equal to his initial endowment $e^{(i)}$. The wealth of any non-young investor $(i, j) \in \mathbb{I}, j<J$ at time $t$ corresponds to the value of his portfolio $\left(x_{t-1}^{(i, j+1)}, y_{t-1}^{(i, j+1)}\right)$ from the previous period at prices of period $t$. We therefore set

$$
w_{t}^{(i j)}= \begin{cases}e^{(i)} & j=J \\ R y_{t-1}^{(i, j+1)}+p_{t}^{\top} x_{t-1}^{(i, j+1)} & j=1, \ldots, J-1\end{cases}
$$

for initial wealth in period $t$. Note that the wealth of a non-young investor $(i, j), j<J$ depends on prices.

In order to obtain explicit demand schedules, we make specific assumptions regarding investors' preferences and beliefs. Investors beliefs in any period $t$ are assumed to be given by multivariate a normal distribution for future prices $p_{t+1}, \ldots, p_{t+j} \in \mathbb{R}^{K}$. Let $\mathcal{M}_{K j}$, denote the set of all symmetric, positive definite $(K j) \times(K j)$ matrices. Recall that a (multivariate) normal distribution with parameters $(\mu, \Sigma) \in \mathbb{R}^{K j} \times \mathcal{M}_{K j}$ is given

${ }^{2}$ Noise traders will be thought of as traders whose portfolio decisions are not captured by a standard microeconomic decision model. Alternative interpretations as those of De Long, Shleifer, Summers \& Waldmann (1990, p. 709) apply as well. 
by the density function

$$
f_{K j}(q ; \mu, \Sigma):=(2 \pi)^{-\frac{N}{2}}[\operatorname{det} \Sigma]^{-\frac{1}{2}} \exp \left\{-\frac{1}{2}(q-\mu)^{\top} \Sigma^{-1}(q-\mu)\right\}, \quad q \in \mathbb{R}^{K j},
$$

cf. Tong (1990). As a further technical restriction, let $\mathcal{M}_{K j}^{\star} \subset \mathcal{M}_{K j}$ denote the class of all symmetric, positive definite $K j \times K j$ matrices which satisfy a certain invertibility condition which will be made explicit in Assumption 4, Appendix A. We are now ready to specify the assumptions on investors who maximize utility of terminal wealth.

\section{Assumption 2}

Preferences and beliefs of investor $(i, j) \in \mathbb{I}$ are characterized by the following:

1. Preferences of an investors of type $i$ are described by an exponential utility function

$$
u\left(w ; a^{(i)}\right):=-\exp \left\{-a^{(i)} w\right\}, \quad w \in \mathbb{R},
$$

where $a^{(i)}>0$ denotes risk-aversion.

2. The subjective beliefs of investor $(i, j) \in \mathbb{I}$ at time $t$ regarding prices $p_{t+1}, \ldots, p_{t+j}$ are given by a normal distribution on $\mathbb{R}^{K j}$ with density function of the form (2) described by the first two moments

$$
\mu_{t}^{(i j)}:=\left(\begin{array}{c}
\mu_{t, t+1}^{(i)} \\
\vdots \\
\mu_{t, t+j}^{(i)}
\end{array}\right) \in \mathbb{R}^{K j}, \quad \Sigma_{t}^{(i j)}:=\left[\begin{array}{ccc}
\Sigma_{t, 11}^{(i)} & \ldots & \Sigma_{t, 1 j}^{(i)} \\
\vdots & \ddots & \vdots \\
\Sigma_{t, j 1}^{(i)} & \ldots & \Sigma_{t, j j}^{(i)}
\end{array}\right] \in \mathcal{M}_{K j}^{\star} .
$$

Here, $\mu_{t, t+s}^{(i j)}:=\mathbb{E}_{t}^{(i j)}\left[p_{t+s}\right]$ denotes investor $(i, j)$ 's subjective mean value for prices $p_{t+s}, s=1, \ldots, j$ conditional on information available at time $t$ corresponding to the density $f_{K j}\left(\cdot, \mu_{t}^{(i j)}, \Sigma_{t}^{(i j)}\right)$. The matrix

$$
\Sigma_{t, s s^{\prime}}^{(i j)}:=\mathbb{E}_{t}^{(i j)}\left[\left(p_{t+s}-\mathbb{E}_{t}^{(i j)}\left[p_{t+s}\right]\right)\left(p_{t+s^{\prime}}-\mathbb{E}_{t}^{(i j)}\left[p_{t+s^{\prime}}\right]\right)^{\top}\right]
$$

denotes investor $(i, j)$ 's subjective conditional variance-covariance matrix between the prices $p_{t+s}, p_{t+s^{\prime}}, s, s^{\prime}=1, \ldots, j$ corresponding to $f_{K j}\left(\cdot, \mu_{t}^{(i j)}, \Sigma_{t}^{(i j)}\right)$.

Assumption 2 states that all investors of the same type are characterized by the same risk aversion. The subjective beliefs of investor $(i, j) \in \mathbb{I}$ at time $t$ are parameterized by subjective means $\mu_{t}^{(i j)} \in \mathbb{R}^{K j}$ and subjective variance-covariance-matrices $\Sigma_{t}^{(i j)} \in \mathcal{M}_{K j}^{\star}$ for future prices. Assumption 4, Appendix A shows that the restriction to $\mathcal{M}_{K j}^{\star}$ is trivially satisfied if $\Sigma_{s s^{\prime}}^{(i)}=0$ for $s \neq s^{\prime}$ and hence if correlations between prices of different periods are sufficiently small. For simplicity, we presume in Assumption 2 that any nonyoung investor $(i, j) \in \mathbb{I}$ with a planning horizon $j<J$ holds the same expectations for prices $p_{t+1}, \ldots, p_{t+j}$ as the young investor $(i, J)$. Formally, this means that her 
beliefs are given by the marginal distributions ${ }^{3}$ of the respective young investor $(i, J)$. Economically, this assumption can be justified by presuming that all investors of type $i$ employ the same financial mediator. As a consequence, expectations of all investors at time $t$ are completely described by the moments of the respective young cohort. Notice that all subjective moments $\left(\mu_{t}^{(i j)}, \Sigma_{t}^{(i j)}\right)$ are by assumption $\mathcal{F}_{t-1}$ measurable.

Consider the portfolio choice problem faced by an arbitrary investor $(i, j) \in \mathbb{I}$ in an arbitrary but fixed period, say $t=0$. For simplicity of notation, we write his beliefs (4) as

$$
\mu:=\left(\begin{array}{c}
\mu_{1} \\
\vdots \\
\mu_{j}
\end{array}\right) \in \mathbb{R}^{K j} \quad \Sigma:=\left[\begin{array}{ccc}
\Sigma_{11} & \ldots & \Sigma_{1 j} \\
\vdots & \ddots & \vdots \\
\Sigma_{j 1} & \ldots & \Sigma_{j j}
\end{array}\right] \in \mathcal{M}_{K j}^{\star}
$$

and suppress type indices and the time index referring to the decision period $t=0$ for a moment. Given parametric prices $p$ and initial wealth $w$ defined by (1) assume that the investor chooses a self-financing trading strategy

$$
H=\left(x_{0}, y_{0}, \ldots, x_{j-1}, y_{j-1}\right)
$$

consisting of a list of portfolios $\left(x_{0}, y_{0}\right) \in \mathbb{R}^{K} \times \mathbb{R}$ and planned portfolios

$$
x_{s}=x_{s}\left(p_{1}, \ldots, p_{s}\right) \in \mathbb{R}^{K}, \quad y_{s}=y_{s}\left(p_{1}, \ldots, p_{s}\right) \in \mathbb{R}, \quad s=1, \ldots, j-1,
$$

such that for each possible realization of prices $p_{1}, \ldots, p_{s}$, the following budget conditions hold:

$$
\begin{aligned}
y_{0}+p^{\top} x_{0} & =w \\
y_{s}+p_{s}^{\top} x_{s} & =R y_{s-1}+p_{s}^{\top} x_{s-1}, \quad s=1, \ldots, j-1, \\
W_{j} & =R y_{j-1}+p_{j}^{\top} x_{j-1} .
\end{aligned}
$$

Observe that for each $s=1, \cdots, j-1$, planned portfolios $\left(x_{s}, y_{s}\right)$ are mappings that depend on prices $p_{1}, \ldots, p_{s}{ }^{4}$

Let $\mathscr{H}(p, w)$ denote the set of all self-financing strategies satisfying (6) with parametric prices $p$ and initial wealth $w$ at time $t=0$. Setting $p_{1}^{s}:=\left(p_{1}, \ldots, p_{s}\right)$, the choice of a particular strategy $H \in \mathscr{H}(p, w)$ induces a random variable $W_{j}\left(H, p_{1}^{j}\right):=R y_{j-1}\left(p_{1}^{j-1}\right)+$ $p_{j}^{\top} x_{j-1}\left(p_{1}^{j-1}\right)$ which describes terminal wealth attained at the end of period $j$. Assuming that given his beliefs $(\mu, \Sigma) \in \mathbb{R}^{K j} \times \mathcal{M}_{K j}^{\star}$, parametric prices $p \in \mathbb{R}^{K}$ and wealth $w \in \mathbb{R}$ the investor maximizes the expected utility of terminal wealth his optimization problem at $t=0$ reads

$$
\max \left\{\int_{\mathbb{R}^{K j}} u\left(W_{j}\left(H, p_{1}^{j}\right) ; a^{(i)}\right) f_{K j}\left(p_{1}^{j}, \mu, \Sigma\right) d p_{1}^{j} \mid H \in \mathscr{H}(p, w)\right\}
$$

\footnotetext{
${ }^{3}$ Mathematically, the subjective probability distribution of a non-young investor $(i, j)$ is the projection of the probability distribution of the corresponding young investor $(i, J)$. By the properties of the multivariate normal distribution (e.g., see Tong 1990) this distribution is again normal with corresponding projected moments.

4 This definition of a self-financing trading strategy is consistent with Pliska (1997) who defines a trading strategy as an adapted stochastic process.
} 
Note that the investor is allowed to update beliefs and reoptimize planned portfolio decisions in any subsequent period. A solution to the optimization problem (7) will determine the investors' individual asset demand functions at time $t=0$ as a function of prices, wealth and beliefs. Using a dynamic programming approach we show in Appendix A that the restriction of the subjective covariance-variance matrices $\Sigma$ to the set $\mathcal{M}_{K j}^{\star} \subset \mathcal{M}_{K j}$ suffices to obtain well-defined asset demand functions. These demand functions are given in the following Theorem.

\section{Theorem 1}

Let Assumption 2 be satisfied. Then for each planning horizon $j=1, \ldots, J$ the investor's asset demand function derived from (7) given her beliefs $(\mu, \Sigma) \in \mathbb{R}^{K j} \times \mathcal{M}_{K j}^{\star}$ and risk aversion $a^{(i)}$ takes the form:

$$
\varphi^{(i j)}(p, \mu, \Sigma):=\frac{1}{R^{j-1} a^{(i)}} \Pi_{j}^{\top} \Sigma^{-1}\left[\mu-\Pi_{j} R p\right], \quad p \in \mathbb{R}^{K} .
$$

where $\Pi_{j}:=\left[I_{K}, \ldots, R^{j-1} I_{K}\right]^{\top} \in \mathbb{R}^{K j \times K}, j=1, \ldots, J$.

The proof of Theorem 1 is given in Appendix A. Observe that the demand for risky assets (8) is independent of the investor's initial wealth. For a one-period planning horizon $j=1$ and $\left(\mu_{1}, \Sigma_{11}\right) \in \mathbb{R}^{K} \times \mathcal{M}_{K}^{\star}$ Theorem 1 gives

$$
\varphi^{(i 1)}\left(p, \mu_{1}, \Sigma_{11}\right)=\frac{1}{a^{(i)}} \Sigma_{11}^{-1}\left[\mu_{1}-R p\right],
$$

recovering the classical demand function of an investor with linear mean-variance preferences (e.g., see Böhm \& Chiarella 2000). For a two-period planning horizon $j=2$ $(\mu, \Sigma) \in \mathbb{R}^{2 K} \times \mathcal{M}_{2 K}^{\star}$, the demand function (8) reads

$$
\varphi^{(i 2)}(p, \mu, \Sigma)=\frac{1}{R a^{(i)}}\left[I_{K}, R I_{K}\right]\left[\begin{array}{cc}
\Sigma_{11} & \Sigma_{12} \\
\Sigma_{21} & \Sigma_{22}
\end{array}\right]^{-1}\left(\begin{array}{c}
\mu_{1}-R p \\
\mu_{2}-R^{2} p
\end{array}\right) .
$$

On the other hand, if investors assume future prices to be uncorrelated over time, i.e. $\Sigma_{s s^{\prime}}=\Sigma_{s^{\prime} s}^{\top}=0$ for all $s \neq s^{\prime}$, then the demand function (10) takes the form

$$
\varphi^{(i j)}(p, \mu, \Sigma)=\frac{1}{a^{(i)} R^{j-1}} \sum_{s=1}^{j}\left(\frac{1}{R^{2(s-1)}} \Sigma_{s s}\right)^{-1}\left[\frac{1}{R^{s-1}} \mu_{s}-R p\right] .
$$

In this case the asset demand function is the sum of $j$ asset demand functions of the form (9) with adjusted risk aversion $a^{(i)} R^{j-1}$ and discounted moments $\left(\frac{1}{R^{s-1}} \mu_{s}, \frac{1}{R^{2(s-1)}} \Sigma_{s s}\right)$.

In the sequel we assume that in each period $t \in \mathbb{N}$ each investor $(i, j) \in \mathbb{I}, j>0$, solves an optimization problem of the form (7) given her beliefs $\left(\mu_{t}^{(i j)}, \Sigma_{t}^{(i j)}\right) \in \mathbb{R}^{(K j)} \times \mathcal{M}_{K j}^{\star}$, her initial wealth (1) of period $t$ and risk aversion $a^{(i)}>0$. By Theorem 1 investor $(i, j)$ 's demand function at time $t$ is

$$
\varphi^{(i j)}\left(p, \mu_{t}^{(i j)}, \Sigma_{t}^{(i j)}\right)=\frac{1}{R^{j-1} a^{(i)}} \Pi_{j}^{\top} \Sigma_{t}^{(i j)-1}\left[\mu_{t}^{(i j)}-\Pi_{j} R p\right], \quad p \in \mathbb{R}^{K} .
$$

Setting for each $j>0$

$$
B_{t}^{(i j)}=\left[B_{t, 1}^{(i j)}, \ldots, B_{t, j}^{(i j)}\right]:=\Pi_{j}^{\top} \Sigma_{t}^{(i j)-1} \in \mathbb{R}^{K \times K j}
$$


with $B_{t, s}^{(i j)} \in \mathbb{R}^{K \times K}$ and

$$
C_{t}^{(i j)}:=\Pi_{j}^{\top} \Sigma_{t}^{(i j)-1} \Pi_{j} \in \mathbb{R}^{K \times K},
$$

the asset demand function (11) takes the form

$$
\varphi^{(i j)}\left(p, \mu_{t}^{(i j)}, \Sigma_{t}^{(i j)}\right)=\frac{1}{a^{(i)} R^{j-1}}\left(\sum_{s=1}^{j} B_{t, s}^{(i j)} \mu_{t, t+s}^{(i)}-C_{t}^{(i j)} R p\right), \quad p \in \mathbb{R}^{K} .
$$

The consequences of Theorem 1 seem quite remarkable. It establishes a structural equivalence between the demand functions of investors with a one period planning horizon and investors with a multiperiod planning horizon of arbitrary finite length. In principle, subjective expectations of an investor with a multiperiod planning horizon can always be transformed in such a way that his demand behavior is indistinguishable from the behavior of an investor with a one-period planning horizon. Despite this fact, it turns out that many interesting implications arise due to the intrinsic heterogeneity of different planning horizons some of which are addressed in the present paper.

\subsection{Price formation}

In order to determine market clearing prices, let $\bar{x} \in \mathbb{R}_{+}^{K}$ denote the total stock of risky assets. Market clearing in period $t$ requires the existence of a price vector $p_{t} \in \mathbb{R}^{K}$ such that aggregate demand including noise traders equals the total stock of risky assets. Given the individual demand functions (11) for risky assets and the quantity of noise traders $\xi_{t}$, the market-clearing condition of period $t$ reads

$$
\sum_{(i, j) \in \mathbb{I}} \frac{1}{R^{j-1} a^{(i)}}\left(\Pi_{j}^{\top} \Sigma_{t}^{(i j)-1} \mu_{t}^{(i j)}-\left(\Pi_{j}^{\top} \Sigma_{t}^{(i j)-1} \Pi_{j}\right) R p\right)+\xi_{t} \stackrel{!}{=} \bar{x} .
$$

Solving for $p_{t}$, given any list of subjective beliefs $\left(\mu_{t}^{(i j)}, \Sigma_{t}^{(i j)}\right)_{(i, j) \in \mathbb{I}}$ and $\xi_{t}$, the market clearing prices are defined by a map

$$
p_{t}=S\left(\left(\mu_{t}^{(i j)}, \Sigma_{t}^{(i j)}\right)_{(i, j) \in \mathbb{I}}, \xi_{t}\right):=\sum_{(i, j) \in \mathbb{I}} A_{t}^{(i j)} \mu_{t}^{(i j)}-A_{t}\left[\bar{x}-\xi_{t}\right],
$$

where $^{5}$

$$
\begin{aligned}
& A_{t}:=\frac{1}{R}\left[\sum_{(i, j) \in \mathbb{I}} \frac{1}{a^{(i)} R^{j-1}} \Pi_{j}^{\top} \Sigma_{t}^{(i j)-1} \Pi_{j}\right]^{-1}=\frac{1}{R}\left[\sum_{(i, j) \in \mathbb{I}} \frac{1}{a^{(i)} R^{j-1}} C_{t}^{(i j)}\right]^{-1}, \\
& A_{t}^{(i j)}:=\frac{1}{a^{(i)} R^{j-1}} A_{t} \Pi_{j}^{\top} \Sigma_{t}^{(i j)-1}=\frac{1}{a^{(i)} R^{j-1}} A_{t} B_{t}^{(i j)}
\end{aligned}
$$

Portfolio holdings of investors $(i, j) \in \mathbb{I}$ after trading in period $t$ are given by

$$
\begin{aligned}
x_{t}^{(i j)} & =\varphi^{(i j)}\left(p_{t}, \mu_{t}^{(i j)}, \Sigma_{t}^{(i j)}\right), \\
y_{t}^{(i j)} & = \begin{cases}e^{(i)}-p_{t}^{\top} x_{t}^{(i j)}, & \text { if } j=J, \\
R y_{t-1}^{(i, j+1)}+p_{t}^{\top}\left[x_{t-1}^{(i, j+1)}-x_{t}^{(i j)}\right], & \text { if } j=1, \cdots, J-1 .\end{cases}
\end{aligned}
$$

${ }^{5}$ Note that since all $\Pi_{j}$ have rank $K$, all $\Pi_{j}^{\top} \Sigma_{t}^{(i j)-1} \Pi_{j}$ are positive-definite and hence invertible. Since the sum of positive definite matrices is again positive definite, $A_{t}$ and all $A_{t}^{(i j)}$ are well-defined. 
The temporary equilibrium map (16) defines the economic law $S$ for our multiperiod version of the CAPM which determines market-clearing prices in each trading period as a function of agents' expectations for future prices. The map $S$ is of the Cobwebtype since it contains essentially price forecasts as arguments. Since these expectations refer to future periods $t+1, \ldots, t+J$, the law contains an expectational lead of length $J$. Notice that all coefficient matrices $A_{t}^{(i j)}$ and $A_{t}$ are $\mathcal{F}_{t-1}$ measurable such that the uncertainty of the price $p_{t}$ rests solely on the noise trader demand $\xi_{t}$. This generalizes the case with heterogeneous investors with a one-period planning horizon in Wenzelburger (2004). In the present setup, heterogeneity enters through possibly diverse beliefs as well as through different planning horizons of investors belonging to different cohorts.

\section{Homogeneous expectations}

In the following section we will show that the heterogeneous structure of the price law (16) is maintained even if expectations of investors are homogeneous. The latter assumption allows us to isolate the impact of different planning horizons on portfolios and prices. Consider therefore the case with homogeneous expectations where the beliefs of investor $(i, j) \in \mathbb{I}$ are independent of his type $i$ and may depend only on the length $j$ of his planning horizon. This implies in particular that all investors within one cohort hold identical expectations. In the sequel we will therefore write $\left(\mu_{t}^{(j)}, \Sigma_{t}^{(j)}\right) \in \mathbb{R}^{K j} \times$ $\mathcal{M}_{K j}^{\star}$, for the beliefs of cohort $j, j=1, \ldots, J$ in period $t$ instead of $\left(\mu_{t}^{(i j)}, \Sigma_{t}^{(i j)}\right)$. It is straightforward to see that the demand functions of any two investors $(i, j),\left(i^{\prime}, j\right) \in \mathbb{I}$ with homogeneous expectations satisfy the relation

$$
\varphi^{(i j)}\left(p, \mu_{t}^{(j)}, \Sigma_{t}^{(j)}\right)=\frac{1}{a^{(i)} R^{j-1}} \Pi_{j}^{\top} \Sigma_{t}^{(j)-1}\left[\mu_{t}^{(j)}-\Pi_{j} R p\right]=\frac{a^{\left(i^{\prime}\right)}}{a^{(i)} R^{j-1}} \varphi^{\left(i^{\prime} j\right)}\left(p, \mu_{t}^{(j)}, \Sigma_{t}^{(j)}\right)
$$

for all $p \in \mathbb{R}_{+}^{K}$. The demand functions for risky shares of investors belonging to the same cohort are thus collinear by a factor determined by the possibly different risk aversions $a^{(i)}$ and $a^{\left(i^{\prime}\right)}$. This implies that the proportions of shares held by investors of the same cohort are identical.

Let $\alpha:=\left(\frac{1}{a^{(1)}}+\cdots+\frac{1}{a^{(I)}}\right)>0$ denote the aggregate risk tolerance, $p_{t}$ be the marketclearing price in period $t$, and

$$
x_{t}^{(j)}:=\sum_{i=1}^{I} \varphi^{(i j)}\left(p_{t}, \mu_{t}^{(j)}, \Sigma_{t}^{(j)}\right)=\frac{\alpha}{R^{j-1}} \Pi_{j}^{\top} \Sigma_{t}^{(j)-1}\left[\mu_{t}^{(j)}-\Pi_{j} R p_{t}\right]
$$

denote the aggregate generational portfolio held by cohort $j$ after trading in period $t$. This proves the following result.

\section{Theorem 2}

Under homogeneous expectations, the risky portfolio $x_{t}^{(i j)}$ held by an investor $(i, j) \in \mathbb{I}$ after trading in period $t \in \mathbb{N}$ is given by a constant share of the aggregate generational portfolio (19) of cohort $j$, such that

$$
x_{t}^{(i j)}=\frac{\frac{1}{a^{(i)}}}{\alpha} x_{t}^{(j)} .
$$


This share is determined by the individual risk tolerance $\frac{1}{a^{(i)}}$ relative to the aggregate risk tolerance $\alpha$.

Theorem 2 is a generalization of the famous capital market line result from classical CAPM theory to the case with multiperiod planning horizons. Under homogeneous expectations, investors will hold a multiple of a generational portfolio rather than the market portfolio $\bar{x}$. As a consequence of Theorem 1 the generational portfolios corresponding to different planning horizons will, in general, not be collinear. Therefore, even under homogeneous expectations, planning horizons of distinct lengths will lead to structurally distinct portfolio holdings.

\section{Unbiased forecasting rules}

The price law (16) determines market clearing prices in each period given the beliefs of all investors and the demand of noise traders. To obtain a complete description of the dynamic evolution of prices and portfolios we need to specify how investors form their expectations based on the available information. In this regard, the existence of forecasting rules generating expectations which are rational in some sense is of particular importance which will be studied in this section. Since by virtue of Assumption 2 subjective beliefs are characterized by the corresponding first two moments, the notion of rational expectations employed here is that the first two moments of the price process induced by (16) are correctly predicted for all times $t$ conditional on the available information. To this end, the following two sections will develop forecasting rules which provide correct predictions of first and second moments of the price process.

Following Wenzelburger (2001, 2004), we develop unbiased forecasting rules that generate rational expectations for investors of type $I$. The notion of rational expectations used here requires that forecast must be unbiased in the sense that in each trading period the subjective expected values and variance-covariance matrix for future prices coincide with the respective true conditional moments.

\section{$4.1 \quad$ Unbiased first moments}

Assume that investors of type $I$ use a no-updating forecasting rule of the following form. The idea of such a forecasting rule is that in any period $t$, the first $J-1$ forecasts will not be updated such that

$$
\mu_{t, t+j}^{(I)}=\mu_{t-1, t+j}^{(I)}, \quad j=1, \ldots, J-1 .
$$

Let $\mathbb{E}_{t-t}[\cdot]$ denote the expectations operator taken with respect to the $\sigma$-field $\mathcal{F}_{t-1}$. Since the coefficient matrices $A_{t}^{(i j)}$ and $A_{t}$ in the price law (16) are $\mathcal{F}_{t-1}$ measurable, the idea is to choose $\mu_{t, t+J}^{(I)}$ such that

$$
\mathbb{E}_{t-1}\left[p_{t}\right]=\sum_{(i, j) \in \mathbb{I}} A_{t}^{(i j)} \mu_{t}^{(i j)}-A_{t}\left[\bar{x}-\mathbb{E}_{t-1}\left[\xi_{t}\right]\right] \stackrel{!}{=} \mu_{t-1, t}^{(I)}
$$


Suppose for a moment that the forecasts $\mu_{t, t+J}^{(I)}$ can be chosen such that (21) holds. Then $\mathbb{E}_{t-1}\left[p_{t}-\mu_{t-1, t}^{(I)}\right]=0$ and the no-updating condition implies that the conditional forecast errors of all forecasts $\mu_{t-j, t}^{(I)}, j=1, \ldots, J$ for $p_{t}$ vanish, that is,

$$
\mathbb{E}_{t-1}\left[p_{t}-\mu_{t-j, t}^{(I)}\right]=0, \quad j=1, \ldots, J .
$$

Moreover, by the law of iterated expectations

$$
\mathbb{E}_{t-j}\left[p_{t}-\mu_{t-j, t}^{(I)}\right]=\mathbb{E}_{t-j}\left[\mathbb{E}_{t-1}\left[p_{t}-\mu_{t-j, t}^{(I)}\right]\right]=0, \quad j=1, \ldots, J .
$$

The problem of obtaining unbiased forecasts is therefore reduced to solving (21). This can be achieved as follows. Taking conditional expectations of the market-clearing condition (15), we have

$$
\sum_{(i, j) \in \mathbb{I}} \varphi^{(i j)}\left(\mathbb{E}_{t-1}\left[p_{t}\right], \mu_{t}^{(i j)}, \Sigma_{t}^{(i j)}\right)+\mathbb{E}_{t-1}\left[\xi_{t}\right]-\bar{x}=0
$$

Inserting into (21), it follows that condition (21) is equivalent to

$$
\sum_{(i, j) \in \mathbb{I}} \varphi^{(i j)}\left(\mu_{t-1, t}^{(I)}, \mu_{t}^{(i j)}, \Sigma_{t}^{(i j)}\right)+\mathbb{E}_{t-1}\left[\xi_{t}\right]-\bar{x}=0 .
$$

Let

$$
\zeta_{t}:=\sum_{i=1}^{I} \sum_{j=1}^{J-1} \varphi^{(i j)}\left(\mu_{t-1, t}^{(I)}, \mu_{t}^{(i j)}, \Sigma_{t}^{(i j)}\right)+\mathbb{E}_{t-1}\left[\xi_{t}\right]
$$

denote the expected aggregate portfolio of all investors $(i, j) \in \mathbb{I}$ except investor $(I, J)$. Replacing (23) in condition (22), we see that the condition

$$
\varphi^{(I J)}\left(\mu_{t-1, t}^{(I)}, \mu_{t}^{(I)}, \Sigma_{t}^{(I)}\right)+\zeta_{t}-\bar{x}=0
$$

is equivalent to the original condition (21). Inserting (14) and assuming that the matrix $B_{t, J}^{(I J)}$ defined in (12) is non-singular, we may solve (24) for $\mu_{t, t+J}^{(I)}$ to get

$$
\mu_{t, t+J}^{(I)}=B_{t, J}^{(I J)-1}\left[a^{(I)} R^{J-1}\left(\bar{x}-\zeta_{t}\right)-\sum_{s=1}^{J-1} B_{t, s}^{(I J)} \mu_{t, t+s}^{(I)}+C_{t}^{(I J)} R \mu_{t-1, t}^{(I)}\right] .
$$

An unbiased forecasting rule for investors of type $I$ is thus given by

$$
\left\{\begin{aligned}
\mu_{t, t+j}^{(I)} & =\mu_{t-1, t+j}^{(I)}, \quad j=1, \ldots, J-1 . \\
\mu_{t, t+J}^{(I)} & =\psi_{\star}^{(I)}\left(\mu_{t-1}^{(I)}, \Sigma_{t}^{(I J)}, \zeta_{t}\right) \\
& :=B_{t, J}^{(I J)-1}\left[a^{(I)} R^{J-1}\left(\bar{x}-\zeta_{t}\right)-\sum_{s=1}^{J-1} B_{t, s}^{(I J)} \mu_{t-1, t+s}^{(I)}+C_{t}^{(I J)} R \mu_{t-1, t}^{(I)}\right] .
\end{aligned}\right.
$$


Inserting the unbiased forecasting rule into the price law (16), we obtain the system of equations

$$
\begin{cases}p_{t} & =\mu_{t-1, t}^{(I)}+A_{t}\left[\xi_{t}-\mathbb{E}_{t-1}\left[\xi_{t}\right]\right] \\ \zeta_{t} & =\sum_{i=1}^{I} \sum_{j=1}^{J-1} \varphi^{(i j)}\left(\mu_{t-1, t}^{(I)}, \mu_{t}^{(i j)}, \Sigma_{t}^{(i j)}\right)+\mathbb{E}_{t-1}\left[\xi_{t}\right] \\ \mu_{t, t+j}^{(I)} & =\mu_{t-1, t+j}^{(I)}, \quad j=1, \ldots, J-1 \\ \mu_{t, t+J}^{(I)} & =B_{t, J}^{(I J)-1}\left[a^{(I)} R^{J-1}\left(\bar{x}-\zeta_{t}\right)-\sum_{s=1}^{J-1} B_{t, s}^{(I J)} \mu_{t-1, t+s}^{(I)}+C_{t}^{(I J)} R \mu_{t-1, t}^{(I)}\right]\end{cases}
$$

that determine the asset prices of period $t$ under rational expectations for investors of type $I$, given the beliefs of all investors $(i, j) \neq(I, J)$. The unbiased forecasting rule $(25)$ is a linear function of the previous forecast $\mu_{t-1, t}^{(I)}$ as well as of the conditional expectation $\mathbb{E}_{t-1}\left[\xi_{t}\right]$ and is independent of previous realizations of prices. Condition (21) is therefore satisfied for all times $t$ if young agents determine their forecast according to (25). Observe that applying the unbiased forecasting rule (25) requires knowledge not only of the previous forecast $\mu_{t-1, t}^{(I)}$ but also of the true conditional expectation $\mathbb{E}_{t-1}\left[\xi_{t}\right]$ of the random variable $\xi_{t}$. In addition to that, knowledge of the expected excess demand of all other investors as well as of the market fundamentals is required to apply (25).

\section{$4.2 \quad$ Perfect second moments}

Building upon the result of the previous section, the following section seeks to develop a forecasting rule which provides correct second moment beliefs of the price process for all times $t$. Extending the ideas from Wenzelburger (2004) to the present multiperiod context, we will derive such a forecasting rule for young investors of type $I$. The projection property of beliefs then implies that all investors of type $I$ will hold correct second moment beliefs.

In the sequel we denote by $\mathbb{V}_{t}[\cdot]$ and $\mathbb{C o v}_{t}[\cdot]$ the (objective) variance and covariance operator conditional on the $\sigma$-algebra $\mathcal{F}_{t}$. The correctness of second moment beliefs requires that for all times $t$ the subjective (block matrix) entries of the matrix $\Sigma_{t}^{(I J)}$ defined in (4) coincide with the corresponding objective moments, that is

$$
\Sigma_{t, j k}^{(I J)} \stackrel{!}{=} \operatorname{Cov}_{t}\left[p_{t+j}, p_{t+k}\right] \quad j, k=1, \ldots, J,
$$

for all times $t$. While perfect forecasting rules for first moments exist generically, more restrictions are required to ensure the existence of a perfect predictor for second moments. To this end, we make the following simplifying assumptions:

\section{Assumption 3}

The following hypotheses are satisfied for all $t$ :

(i) All investors hold constant second moment beliefs such that $\Sigma_{t}^{(i j)} \equiv \Sigma^{(i j)}$ for all $(i, j) \in \mathbb{I}$. 
(ii) Young investors of type I employ the unbiased no-updating forecasting rule $\psi_{\star}^{(I)}$ defined in (25) to predict the first moments of the price process.

(iii) The stochastic process $\left\{\xi_{t}\right\}_{t \in \mathbb{N}}$ is of the $A R(1)$-form

$$
\xi_{t}=h^{(0)}+H^{(1)} \xi_{t-1}+H^{(2)} \varepsilon_{t}
$$

where $h^{(0)} \in \mathbb{R}^{K}$ and $H^{(n)} \in \mathbb{R}^{K \times K}, n=1,2$, are both non-singular and the innovation process $\left\{\varepsilon_{t}\right\}_{t \in \mathbb{N}}$ is a sequence of iid random variables.

Let $\eta_{t}:=\xi_{t}-\mathbb{E}_{t-1}\left[\xi_{t}\right]=H^{(2)} \varepsilon_{t}$. Then the innovation process $\left\{\epsilon_{t}\right\}_{t \in \mathbb{N}}$ in Assumption 3 (iii) implies that for all times $t$ and all $j, k>0$

$$
\operatorname{Cov}_{t}\left[\eta_{t+j}, \eta_{t+k}\right]= \begin{cases}H^{(2)} H^{(2) \top} & \text { for } j=k, \\ 0 & \text { for } j \neq k .\end{cases}
$$

Since $H^{(2)}$ is non-singular, $V_{\eta}:=H^{(2)} H^{(2) \top}$ is positive definite and symmetric.

As a consequence of Assumption $3(i)$, the matrices defined in (17) will be constant over time. In particular

$$
A_{t} \equiv A:=\frac{1}{R}\left[\sum_{i=1}^{I} \sum_{j=1}^{J} \frac{R^{1-j}}{a^{(i)}} \Pi_{j}^{\top} \Sigma^{(i j)-1} \Pi_{j}\right]^{-1}
$$

By Assumption $3(i i)$, prices at time $t$ are then determined by

$$
p_{t}=\mu_{t-1, t}^{(I)}+A \eta_{t}
$$

Recall from (20) that each forecast $\mu_{t-1, t}^{(I)}=\mu_{t-J, t}^{(I)}$ is $\mathcal{F}_{t-J}$-measurable. This together with equations (30) shows that the objective second moments of the price process take the form

$$
\operatorname{Cov}_{t}\left[p_{t+j}, p_{t+k}\right]=A \operatorname{Cov}_{t}\left[\eta_{t+j}, \eta_{t+k}\right] A, \quad j, k=1, \ldots, J .
$$

It follows from (28) that for all $j, k=1, \ldots, J$,

$$
\operatorname{Cov}_{t}\left[p_{t+j}, p_{t+k}\right]= \begin{cases}A V_{\eta} A & \text { for } j=k, \\ 0 & \text { for } j \neq k .\end{cases}
$$

It is readily seen from equation (32) that correlations between prices of distinct periods are zero while the variance-covariance matrix of prices in any period remains constant over time. This implies that the correct variance-covariance matrix for investors of type $I$ must be of the form

$$
\Sigma^{(I J)}=\left[\begin{array}{ccc}
\bar{\Sigma}^{(I)} & \cdots & 0 \\
\vdots & \ddots & \vdots \\
0 & \cdots & \bar{\Sigma}^{(I)}
\end{array}\right]
$$


where $\bar{\Sigma}^{(I)}$ is some $K \times K$ matrix. It follows from (27) and (32) that $\bar{\Sigma}^{(I)}$ must satisfy the equation

$$
A^{-1} \bar{\Sigma}^{(I)} A^{-1} \stackrel{!}{=} V_{\eta}
$$

The existence of a variance covariance matrix which provides correct second moment beliefs for investors of type $I$ is thus reduced to finding a symmetric and positive definite solution to (34). Setting

$$
\begin{aligned}
C & :=R \sum_{i=1}^{I-1} \sum_{j=1}^{J} \frac{1}{a^{(i)} R^{j-1}} \Pi_{j}^{\top} \Sigma^{(i j)-1} \Pi_{j} \\
\rho & :=\sum_{j=1}^{J} \sum_{s=1}^{j} R^{2 s-j}
\end{aligned}
$$

the matrix $A^{-1}$ from (29) may be decomposed into

$$
A^{-1}=C+\frac{\rho}{a^{(I)}} \bar{\Sigma}^{(I)-1} .
$$

Inserting (37), condition (34) takes the form

$$
C \bar{\Sigma}^{(I)} C+2 \frac{\rho}{a^{(I)}} C+\left(\frac{\rho}{a^{(I)}}\right)^{2} \bar{\Sigma}^{(I)-1}=V_{\eta} .
$$

This shows that the unknown $K \times K$ matrix $\bar{\Sigma}^{(I)}$ is determined by the zeros of the polynomial matrix equation (38). In other words, the solutions to (38) provide the block matrix entries of the correct variance-covariance matrix (33).

\section{Proposition 1}

Let Assumption 3 be satisfied. Assume, in addition, that the following holds true.

(i) The matrix $H^{(2)}$ from Assumption 3 (iii) is of the form $H^{(2)}=\sigma_{\eta} I_{K}$, such that $V_{\eta}=H^{(2)} H^{(2) \top}=\sigma_{\eta}^{2} I_{K}$.

(ii) Let $\lambda_{1}, \ldots, \lambda_{K}$ denote the eigenvalues of the positive definite matrix $C^{-1}$ defined in (35) such that

$$
C^{-1}=O^{\top} \operatorname{diag}\left(\lambda_{1}, \ldots, \lambda_{K}\right) O,
$$

and assume that these are sufficiently large such that $\lambda_{k}>\frac{4 \rho}{a^{(I)} \sigma_{\eta}^{2}}$, where $\bar{v}^{(I)}$ is defined in (36).

Then any $K \times K$ matrix

$$
\bar{\Sigma}^{(I)}:=O^{\top} \operatorname{diag}\left(\lambda_{1}^{\Sigma}, \ldots, \lambda_{K}^{\Sigma}\right) O,
$$

with eigenvalues of the form

$$
\lambda_{k}^{\Sigma}=\frac{\rho \lambda_{k}}{a^{(I)}}\left[\left(\frac{a^{(I)} \lambda_{k} \sigma_{\eta}^{2}}{2 \rho}-1\right) \pm \sqrt{\left(\frac{a^{(I)} \lambda_{k} \sigma_{\eta}^{2}}{2 \rho}-1\right)^{2}-1}\right], \quad k=1, \ldots, K
$$

is a solution to (38). As a consequence, any variance-covariance matrix $\Sigma^{(I J)} \in \mathcal{M}_{K J}$ as given in (33) with block matrix entries $\bar{\Sigma}^{(I)}$ provides correct second moment beliefs for young investors of type $I$ for all times $t$. 
Proof of Proposition 1. Set

$$
B:=\frac{a^{(I)}}{\rho} V_{\eta} C^{-1}-2 I_{K} \quad \text { and } \quad D:=\frac{a^{(I)}}{\rho} C \bar{\Sigma}^{(I)} .
$$

The condition (38) becomes a matrix equation of the form

$$
D+D^{-1} \stackrel{!}{=} B
$$

Since $B$ is symmetric and positive definite, it is diagonalizable such that

$$
B=O^{\top} \operatorname{diag}\left(\lambda_{1}^{B}, \ldots, \lambda_{K}^{B}\right) O,
$$

with some orthogonal matrix $O$ and eigenvalues

$$
\lambda_{k}^{B}=\frac{a^{(I)} \lambda_{k}}{\rho} \sigma_{\eta}^{2}-2>0, \quad k=1, \ldots, K .
$$

To solve (40), choose a symmetric matrix of the form

$$
D=O^{\top} \operatorname{diag}\left(\kappa_{1}, \ldots, \kappa_{K}\right) O .
$$

Recall that $O^{\top} O=I_{K}$. Inserting (41), the matrix equation (40) is transformed into $K$ scalar equations for eigenvalues of $D$ which take the form

$$
\kappa_{k}+\frac{1}{\kappa_{k}}=\lambda_{k}^{B}, \quad k=1, \ldots, K .
$$

Replacing the $\lambda_{k}^{B}$, the solutions to (42) are

$$
\kappa_{k}=\left(\frac{a^{(I)} \lambda_{k} \sigma_{\eta}^{2}}{2 \rho}-1\right) \pm \sqrt{\left(\frac{a^{(I)} \lambda_{k} \sigma_{\eta}^{2}}{2 \rho}-1\right)^{2}-1}, \quad k=1, \ldots, K .
$$

Hence the solutions $\bar{\Sigma}^{(I)}$ of (38) must be of the form

$$
\bar{\Sigma}^{(I)}=\frac{\rho}{a^{(I)}} D C^{-1}=O^{\top} \operatorname{diag}\left(\lambda_{1}^{\Sigma}, \ldots, \lambda_{K}^{\Sigma}\right) O
$$

with eigenvalues given in (39).

A special case arises if all young investors have identical (unbiased) second moment beliefs of the form (33). In this case we have from (29)

$$
A=\frac{1}{\alpha \rho} \bar{\Sigma}^{(I)}, \quad \text { where } \quad \alpha=\sum_{i=1}^{I} \frac{1}{a^{(i)}} .
$$

Substituting (43) into (34) yields the following.

\section{Proposition 2}

Let Assumption 3 be satisfied. In addition, assume that all young investors have homogeneous second moment beliefs of the form (33). Then the matrix $\Sigma^{(I J)}$ defined in (33) with block matrix entries

$$
\bar{\Sigma}^{(I)}=(\alpha \rho)^{2} V_{\eta}^{-1}
$$

and $\rho$ given by (36) provides correct second moment beliefs of the price process for all times $t$. In the case of a 2-period planning horizon $(J=2)(44)$ reads

$$
\bar{\Sigma}^{(I)}=\alpha^{2}\left(1+R+R^{2}\right)^{2} V_{\eta}^{-1} .
$$




\section{Evolution of Asset Prices}

\subsection{Chartists and Fundamentalists}

In this section we shall set up the dynamics of prices and expectations within a particular scenario in which only two types of investors trade on the market, i.e., $I=2$. Investors of type $i=1$ will henceforth be called chartists while investors of type $i=2$ will be referred to as fundamentalists. The main difference between these two types of investors lies in their formation of expectations. Chartists base their forecasts for future prices on a weighted average of the $L$ previous observations of prices such that their forecast at time $t$ for the price vector $p_{t+j}$ takes the form

$$
\mu_{t, t+j}^{(1)}=\sum_{l=1}^{L} D^{(l j)} p_{t-l}, \quad j=1, \ldots, J,
$$

where $D^{(l j)} \in \mathbb{R}^{K \times K}$. Fundamentalists are assumed to use the unbiased forecasting rule (25) developed in section 4.1.

We maintain Assumption 3 and, in addition, assume for simplicity that investors of either type with planning horizon $j>0$ use a constant variance-covariance matrix of the diagonal form

$$
\Sigma_{t}^{(i j)} \equiv \Sigma^{(i j)}:=\left[\begin{array}{ccc}
\bar{\Sigma}^{(i)} & \cdots & 0 \\
\vdots & \ddots & \vdots \\
0 & \cdots & \bar{\Sigma}^{(i)}
\end{array}\right] \in \mathcal{M}_{K j}, \quad j=1, \ldots, J, i=1,2
$$

for all times $t$. The matrix $\bar{\Sigma}^{(2)}$ is chosen according to Proposition 1 such that fundamentalists hold correct second moment beliefs of the price process.

Given the particular structure (46) of second moment beliefs the structure of the unbiased forecasting rule (25) greatly simplifies. Using (46) the parameter matrices of the corresponding asset demand functions defined in (12) and (13) take for each $i=1,2$ the simple form

$$
B_{t, s}^{(i j)}:=R^{s-1} \bar{\Sigma}^{(i)-1}, \quad s=1, \ldots, j, j=1, \ldots, J
$$

and

$$
C_{t}^{(i j)}:=\left(\sum_{s=1}^{j} R^{2(s-1)}\right) \bar{\Sigma}^{(i)-1}
$$

for all times $t$. Consequently,

$$
A_{t} \equiv A=\frac{1}{\rho}\left[\frac{1}{a^{(1)}} \bar{\Sigma}^{(1)-1}+\frac{1}{a^{(2)}} \bar{\Sigma}^{(2)-1}\right]^{-1}
$$

for all times $t$. In view of the unbiased forecasting rule (25), the expected aggregate demand is

$$
\zeta_{t}=\frac{1}{a^{(1)}} \bar{\Sigma}^{(1)-1}\left(\sum_{j=1}^{J} \sum_{s=1}^{j} R^{s-j} \mu_{t, t+s}^{(1)}-\rho \mu_{t-1, t}^{(2)}\right)+\mathbb{E}_{t-1}\left[\xi_{t}\right]
$$


Using (47) and (48) together with (49) the unbiased period- $J$ ahead forecast of the fundamentalists at time $t$ becomes

$$
\begin{aligned}
\mu_{t, t+J}^{(2)}= & a^{(2)} \bar{\Sigma}^{(2)}\left(\bar{x}-\mathbb{E}_{t-1}\left[\xi_{t}\right]\right)+\left[\left(\sum_{s=1}^{J} R^{2 s-J}\right) I_{K}+\rho \frac{a^{(2)}}{a^{(1)}} \bar{\Sigma}^{(2)} \bar{\Sigma}^{(1)-1}\right] \mu_{t-1, t}^{(2)} \\
& -\sum_{s=1}^{J-1} R^{s-J} \mu_{t-1, t+s}^{(2)}-\frac{a^{(2)}}{a^{(1)}} \bar{\Sigma}^{(2)} \bar{\Sigma}^{(1)-1} \sum_{j=1}^{J} \sum_{s=1}^{j} R^{s-j} \mu_{t, t+s}^{(1)} .
\end{aligned}
$$

Inserting the chartists' forecasting rule (45), the unbiased forecasting rule (50) becomes a linear function of past forecasts and prices given by

$$
\left\{\begin{array}{l}
\mu_{t, t+j}^{(2)}=\mu_{t-1, t+j}^{(2)}, \quad j=1, \ldots, J-1 \\
\mu_{t, t+J}^{(2)}=\sum_{j=0}^{J-1} E_{j} \mu_{t-1, t+j}^{(2)}+\sum_{l=1}^{L} D_{l} p_{t-l}+a^{(2)} \bar{\Sigma}^{(2)}\left(\bar{x}-\mathbb{E}_{t-1}\left[\xi_{t}\right]\right)
\end{array}\right.
$$

where

$$
\begin{aligned}
E_{0} & :=\left(\sum_{s=1}^{J} R^{2 s-J}\right) I_{K}+\rho \frac{a^{(2)}}{a^{(1)}} \bar{\Sigma}^{(2)} \bar{\Sigma}^{(1)-1}, \\
E_{j} & :=R^{j-J} I_{K}, \quad j=1, \ldots, J-1, \\
D_{l} & :=-\frac{a^{(2)}}{a^{(1)}} \bar{\Sigma}^{(2)} \bar{\Sigma}^{(1)-1} \sum_{j=1}^{J} \sum_{s=1}^{j} R^{s-j} D^{(l s)}, \quad l=1, \ldots, L .
\end{aligned}
$$

Thus we see again that the dynamics of the model are driven by the interaction of fundamentalists' forecasts and actual realizations of prices. Given the structure (46) of second moment beliefs, prices at time $t$ are determined by

$$
p_{t}=\mu_{t-1, t}^{(2)}+\frac{1}{\rho}\left[\frac{1}{a^{(1)}} \bar{\Sigma}^{(1)-1}+\frac{1}{a^{(2)}} \bar{\Sigma}^{(2)-1}\right]^{-1}\left(\xi_{t}-\mathbb{E}_{t-1}\left[\xi_{t}\right]\right) .
$$

Equations (51) and (53) constitute the key elements of the dynamics of the model. Both possess a linear structure with additive noise-terms. As a consequence of this, the evolution of the system is governed by an (affine-)linear stochastic difference equation with additive perturbations.

Setting for each $t$ the state vector $z_{t}:=\left(\mu_{t, t+J}^{(2) \top}, \ldots, \mu_{t, t+1}^{(2) \top}, p_{t}^{\top}, \ldots, p_{t-L+1}^{\top}\right)^{\top} \in \mathbb{R}^{K(J+L)}$ and the vector of perturbations $\epsilon_{t}:=\left(\epsilon_{t}^{(1) \top}, 0^{\top}, \ldots, 0^{\top}, \epsilon_{t}^{(2) \top}, 0^{\top}, \ldots, 0^{\top}\right)^{\top} \in \mathbb{R}^{K(J+L)}$ with $\epsilon_{t}^{(1)}:=a^{(2)} \bar{\Sigma}^{(2)}\left(\bar{x}-\mathbb{E}_{t-1}\left[\xi_{t}\right]\right)$ and $\epsilon_{t}^{(2)}:=A\left(\xi_{t}-\mathbb{E}_{t-1}\left[\xi_{t}\right]\right)$, the dynamics of the system is driven by

$$
z_{t}=M z_{t-1}+\epsilon_{t}
$$


where the $K(J+L) \times K(J+L)$ matrix $M$ takes the form

$$
M:=\left[\begin{array}{cccccc}
E_{J-1} & \cdots & E_{0} & D_{1} & \cdots & D_{L} \\
I_{K} & 0 & \cdots & \cdots & \cdots & 0 \\
0 & \ddots & \ddots & & & \vdots \\
\vdots & \ddots & \ddots & \ddots & & \vdots \\
\vdots & & \ddots & \ddots & \ddots & \vdots \\
0 & \cdots & \cdots & 0 & I_{K} & 0
\end{array}\right]
$$

Equation (54) constitutes a random dynamical system in the sense of Arnold (1998). In the stable case, the long-run behavior of prices and expectations is governed by a stationary stochastic process, random fixed point. In the present case, existence and stability of such a fixed point are determined by the matrix $M$ and hence depends crucially on the risk-free rate of return $R$ as well as on agents' attitude towards risk and chartists' forecasting behavior described by (45).

\section{Conclusions}

We developed a fully explicit and dynamic model of a financial market in which investors with different planning horizons of arbitrary finite length interact. An innovative feature of the models is that investors are allowed to reoptimize their portfolios through the course of time until they leave the market. In addition to possibly diverse beliefs, this model allows to investigate the impact of different planning horizons on asset prices, returns, and portfolios. Introducing the notion of a generational portfolio, we extended the notion of a market portfolio and showed that traders with different planning horizons will generally hold different portfolios even if expectations are homogeneous. To additional issue are on our research agenda. First, the dynamics of asset prices and portfolios thereby allowing investors to switch between different strategies as in Brock \& Hommes $(1997,1998)$ or Chiarella \& He (2002). Second, it would be interesting to know what mean-variance efficient portfolios look alike in the presence of heterogeneous multiperiod planning horizons and whether the results on their performance Böhm \& Wenzelburger (2002) can be generalized. 


\section{A Appendix}

\section{A.1 Proof of Theorem 1}

In this section we solve the decision problem (7) of an arbitrary investor $(i, j) \in \mathbb{I}$ in period $t=0$. All technical prerequisites are collected in the technical Appendix A.2. Since the case with a one-period planning horizon $(j=1)$ is found in the literature (e.g., see Ingersoll 1987) resulting in the demand function (9), we let $j>1$. For notational convenience, the index $(i, j)$ as well as the time index $t=0$ will be omitted in the sequel. Expectations at time $t=0$ for prices $p_{1}, \ldots, p_{j}$ are given by a joint multivariate normal distribution characterized by the first two moments given in (5). Recall to this end that a Gaussian function in $z \in \mathbb{R}^{N}$ with parameters $(c, \mu, \Sigma) \in \mathbb{R}_{++} \times \mathbb{R}^{N} \times \mathcal{M}_{N}$ is a real-valued function

$$
g_{N}(z ; c, \mu, \Sigma):=c \exp \left\{-\frac{1}{2}(z-\mu)^{\top} \Sigma^{-1}(z-\mu)\right\}, \quad z \in \mathbb{R}^{N} .
$$

Setting, $c_{N}(\Sigma):=(2 \pi)^{-\frac{N}{2}}[\operatorname{det} \Sigma]^{-\frac{1}{2}}$, we have the relation

$$
f_{N}(z ; \mu, \Sigma)=g_{N}\left(z ; c_{N}(\Sigma), \mu, \Sigma\right) .
$$

When using Gaussian functions, we shall frequently suppress the dimension index $N$ and omit the parameter $c$ as an argument if $c=1$, writing $g(z ; \mu, \Sigma) \equiv g(z ; 1, \mu, \Sigma)$ to simplify notation.

In order to solve the decision problem, additional restrictions on variance-covariance matrices (5) are required. Setting $\Sigma_{1}^{(j)}:=\Sigma$, for each $s=2, \ldots, j$ we partition the $\operatorname{matrix} \Sigma_{1}^{(s)}$ into $\Sigma_{1}^{(s)}=\left[\begin{array}{cc}\Sigma_{1}^{(s-1)} & \Delta_{s}^{\top} \\ \Delta_{s} & \Sigma_{s s}\end{array}\right]$ with $\Delta_{s}:=\left[\Sigma_{s, 1} \ldots \Sigma_{s, s-1}\right] \in \mathbb{R}^{K \times K(s-1)}$ and define the following parameters

$$
\begin{aligned}
\Sigma_{s} & :=\Sigma_{s s}-\Delta_{s} \Sigma_{1}^{(s-1)-1} \Delta_{s}^{\top} \in \mathcal{M}_{K} \\
\Lambda_{s} & =\left[\Lambda_{s}^{(s-1)} \ldots \Lambda_{s}^{(1)}\right]:=\Delta_{s} \Sigma_{1}^{(s-1)-1} \in \mathbb{R}^{K \times K(s-1)},
\end{aligned}
$$

where $\Lambda_{s}^{(h)} \in \mathbb{R}^{K \times K}, h=1, \ldots, s-1$. Note that each $\Sigma_{s}$ in (58) is well defined, symmetric, and positive definite, cf. Ouellette (1981, Corollary 3.1, p. 208). With the above definitions the following Lemma describes a factorization of the joint probability distribution into marginal and conditional distributions.

\section{Lemma 1}

Let the joint distribution of the random variables $p_{1}, \cdots, p_{j}$ be a normal distribution with moments $(\mu, \Sigma) \in \mathbb{R}^{K j} \times \mathcal{M}_{K j}$ given in (5). Then the following holds:

(1) For each $s=2, \ldots, j$ the conditional distribution of the random variable $p_{s}$ given previous observations $p_{1}, \ldots, p_{s-1}$ is given by a non-singular normal distribution on $\mathbb{R}^{K}$ with moments $\left(\mu_{s \mid s-1}, \Sigma_{s}\right)$, where

$$
\mu_{s \mid s-1}:=\mu_{s}+\Lambda_{s}^{(1)}\left(p_{s-1}-\mu_{s-1}\right)+\ldots+\Lambda_{s}^{(s-1)}\left(p_{1}-\mu_{1}\right) .
$$

and $\Lambda_{s}, \Sigma_{s}$ are defined in (58). 
(2) The marginal distribution of the random variable $p_{1}$ is given by a non-singular normal distribution on $\mathbb{R}^{K}$ with moments $\left(\mu_{1}, \Sigma_{11}\right) \in \mathbb{R}^{K} \times \mathcal{M}_{K}$ given in (5).

Proof. The assertion follows from a repeated application of Theorems 2.4.3 and 2.5.1 given in Anderson (1984), p. 37, see also Tong (1990), Theorem 3.3.4, p. 35.

It will be convenient to introduce some additional notation. For each $s=2, \ldots, j$ set

$$
\mu_{s \mid s-k}:=\mu_{s}-\sum_{n=1}^{k-1} \Lambda_{s}^{(n)} \mu_{s-n}+\sum_{n=k}^{s-1} \Lambda_{s}^{(n)}\left(p_{s-n}-\mu_{s-n}\right), \quad k=1, \ldots, s .
$$

Observe that $\mu_{s \mid s-k}$ is obtained by replacing all prices $p_{s-1}, \ldots, p_{s-k+1}$ in the conditional expectation $\mu_{s \mid s-1}$ with zero vectors. From (58) define for each $n=1, \ldots, j$ the matrices

$$
\Gamma_{n}^{(m)}:= \begin{cases}I_{K} & m=0 \\ R^{m} I_{K}-R^{m-1} \Lambda_{n}^{(1)}-\ldots-R \Lambda_{n}^{(m-1)}-\Lambda_{n}^{(m)} & m=1, \cdots, n-1\end{cases}
$$

To obtain a well-defined asset demand function we impose some restrictions on the variance-covariance matrix $\Sigma$ which are given by the following assumption.

\section{Assumption 4}

All variance-covariance matrices (5) satisfy the following condition. Given the parameters (58) for each $n=2, \cdots, j$ the matrices $\Gamma_{n}^{(1)}:=R I_{K}-\Lambda_{n}^{(1)} \in \mathbb{R}^{K \times K}$ defined in (61) are non-singular and hence invertible. For each $j=1, \ldots, J$, the subset of all variance covariance matrices satisfying (61) is denoted by $\mathcal{M}_{K j}^{\star} \subset \mathcal{M}_{K j}$.

Observe that each set $\mathcal{M}_{K j}^{\star}$ contains all covariance matrices (5) with $\Sigma_{s s^{\prime}}=0$ for $s \neq s^{\prime}$. Hence the set is non-empty for each $j$. In particular, $\mathcal{M}_{K}^{\star}=\mathcal{M}_{K}$ for $j=1$.

Consider now the investor's decision problem (7). To derive a solution, we will employ a dynamic programming approach. This amounts to solving a sequence of $j$ one-stage problems. For each stage $s=0,1, \ldots, j-1$ a one-stage problem is solved given wealth ${ }^{6}$

$$
w_{s}=R y_{s-1}+x_{s-1}^{\top} p_{s}
$$

and realizations of prices $p_{1}, \ldots, p_{s}$ up to time $s$. For the following derivations write $p_{1}^{s}:=\left(p_{1}, \ldots, p_{s}\right)$. For each $s=1, \ldots, j-1$ the conditional distribution of prices $p_{s+1}$ given previous observations $p_{1}, \ldots, p_{s}$ is given by a normal distribution with moments $\left(\mu_{s+1 \mid s}, \Sigma_{s+1}\right)$ given in Lemma 1 (1). Setting $V_{j}\left(w_{j}, p_{1}^{j}\right) \equiv u\left(w_{j} ; a\right)$, the value functions $V_{s}, s=1, \ldots, j-1$ are recursively defined by Bellmann's equation (e.g., see Pliska 1997)

$$
V_{s}\left(w_{s}, p_{1}^{s}\right)=\max _{y+x^{\top} p_{s}=w_{s}}\left\{\int_{\mathbb{R}^{K}} V_{s+1}\left(R y+x^{\top} p, p_{1}^{s}, p\right) f\left(p ; \mu_{s+1 \mid s}, \Sigma_{s+1}\right) d p\right\},
$$

\footnotetext{
6 Since the investor is assumed to choose a self-financing strategy, wealth accumulated at stage $s$ is equal to the return on the investment made in the previous period.
} 
whereas the principle of optimality reads

$$
\begin{aligned}
V_{0}(w, p) & :=\max _{H \in \mathscr{H}(p, w)}\left\{\int_{\mathbb{R}^{K j}} u\left(W_{j}\left(H, p_{1}^{j}\right) ; a\right) f_{K j}\left(p_{1}^{j} ; \mu, \Sigma\right) d p_{1}^{j}\right\} \\
& =\max _{y+x^{\top} p=w}\left\{\int_{\mathbb{R}^{K}} V_{1}\left(R y+x^{\top} p, p\right) f\left(p ; \mu_{1}, \Sigma_{11}\right) d p\right\}
\end{aligned}
$$

We will show below in Proposition 3, Appendix A.2, that the value functions (62) are well defined by computing each $V_{s}$ explicitly. With (63) the portfolio choice problem is reduced to a standard one-stage problem. By Proposition 3 , the value function $V_{1}$ takes the form

$$
V_{1}\left(w_{1}, p_{1}\right)=u\left(w_{1} ; a R^{j-1}\right) g\left(p_{1} ; c_{1}, \vartheta_{1}, \Omega_{1}\right)
$$

where

$$
\begin{aligned}
\Omega_{1} & :=\left(\sum_{n=2}^{j} \Gamma_{n}^{(n-1) \top} \Sigma_{n}^{-1} \Gamma_{n}^{(n-1)}\right)^{-1} \\
\vartheta_{1} & :=\quad \Omega_{1} \sum_{n=2}^{j} \Gamma_{n}^{(n-1) \top} \Sigma_{n}^{-1} \mu_{n \mid 0}
\end{aligned}
$$

and $c_{1}>0$ is a strictly positive constant defined in Proposition 3. By Lemma 2, Appendix A.2 and (57),

$$
g\left(p, c_{1}, \vartheta_{1}, \Omega_{1}\right) f_{K}\left(p, \mu_{1}, \Sigma_{11}\right)=g\left(p, \hat{c}_{1}, \theta_{1}, \Theta_{1}\right)
$$

with

$$
\begin{aligned}
\Theta_{1} & :=\left[\Sigma_{11}^{-1}+\Omega_{1}^{-1}\right]^{-1} \in \mathcal{M}_{K} \\
\theta_{1} & :=\Theta_{1}\left[\Sigma_{11}^{-1} \mu_{1}+\Omega_{1}^{-1} \vartheta_{1}\right] \in \mathbb{R}^{K}
\end{aligned}
$$

and a suitable constant $\hat{c}_{1}>0$. Inserting (64) and (66) into (63), we apply Lemma 3 and Lemma 4 (1) of Appendix A.2 to obtain the solution to (63) as

$$
\left\{\begin{aligned}
x_{0}^{\star} & =\frac{1}{a R^{j-1}} \Theta_{1}^{-1}\left(\theta_{1}-R p\right) \\
y_{0}^{\star} & =w-p^{\top} x_{0}^{\star}
\end{aligned}\right.
$$

We are left to bring (67) into the form stated in the theorem. Using (61) and (65) the matrix $\Theta_{1}^{-1}$ appearing in (67) can be expanded as

$$
\Theta_{1}^{-1}=\Sigma_{11}^{-1}+\sum_{n=2}^{j} \Gamma_{n}^{(n-1) \top} \Sigma_{n}^{-1} \Gamma_{n}^{(n-1)} .
$$

Using $\Pi_{n}=\left[I_{K}, R I_{K}, \ldots, R^{n-1} I_{K}\right]^{\top} \in \mathbb{R}^{K n \times K}$ as previously defined, we can write $\Gamma_{n}^{(n-1)}=-\left[\Lambda_{n}^{(n-1)}, \ldots, \Lambda_{n}^{(1)},-I_{K}\right] \Pi_{n}=-\left[\Lambda_{n},-I_{K}\right] \Pi_{n}$ for each $n=2, \ldots, j$ to obtain

$$
\Gamma_{n}^{(n-1) \top} \Sigma_{n}^{-1} \Gamma_{n}^{(n-1)}=\Pi_{n}^{\top}\left[\begin{array}{c}
\Lambda_{n}^{\top} \\
-I_{K}
\end{array}\right] \Sigma_{n}^{-1}\left[\Lambda_{n},-I_{K}\right] \Pi_{n}, \quad n=2, \ldots, j .
$$


By Ouellette (1981, Theorem 2.7, p.201) the regression parameters $\Sigma_{n}$ and $\Lambda_{n}$ given in (58) take the form

$$
\left[\begin{array}{c}
\Lambda_{n}^{\top} \\
-I_{K}
\end{array}\right] \Sigma_{n}^{-1}\left[\Lambda_{n},-I_{K}\right]=\Sigma_{1}^{(n)-1}-\left[\begin{array}{cc}
\Sigma_{1}^{(n-1)-1} & 0 \\
0 & 0
\end{array}\right], \quad n=2, \ldots, j .
$$

Hence for each $n=2, \ldots, j$,

$$
\Gamma_{n}^{(n-1) \top} \Sigma_{n}^{-1} \Gamma_{n}^{(n-1)}=\Pi_{n}^{\top} \Sigma_{1}^{(n)-1} \Pi_{n}-\Pi_{n-1}^{\top} \Sigma_{1}^{(n-1)-1} \Pi_{n-1} .
$$

Substituting (70) into (68) and noting that trivially $\Pi_{1}^{\top} \Sigma_{1}^{(1)} \Pi_{1}=\Sigma_{11}$ and $\Sigma_{1}^{(j)}=\Sigma$ we see that $(68)$ is a telescope sum and finally obtain

$$
\Theta_{1}^{-1}=\Pi_{j}^{\top} \Sigma^{-1} \Pi_{j}
$$

Using (65) the vector $\theta_{1}$ appearing in (67) can be expanded as

$$
\theta_{1}=\Theta_{1}\left[\Sigma_{11}^{-1} \mu_{1}+\sum_{n=2}^{j} \Gamma_{n}^{(n-1) \top} \Sigma_{n}^{-1} \mu_{n \mid 0}\right]
$$

For each $n=1, \ldots, j$, let $\mu_{1}^{(n)}:=\left(\mu_{1}^{\top}, \ldots, \mu_{n}^{\top}\right)^{\top} \in \mathbb{R}^{K n}$ permitting us to write $\mu_{n \mid 0}=$ $\mu_{n}-\Lambda_{n}^{(1)} \mu_{n-1}-\ldots-\Lambda_{n}^{(n-1)} \mu_{1}=-\left[\Lambda_{n},-I_{K}\right] \mu_{1}^{(n)}$. Writing $\Gamma_{n}^{(n-1)}=-\left[\Lambda_{n},-I_{K}\right] \Pi_{n}$ as above and using (69) yields for each $n=2, \ldots, j$,

$$
\begin{aligned}
\Gamma_{n}^{(n-1) \top} \Sigma_{n}^{-1} \mu_{n \mid 0} & =\Pi_{n}^{\top}\left[\begin{array}{c}
\Lambda_{n}^{\top} \\
-I_{K}
\end{array}\right] \Sigma_{n}^{-1}\left[\Lambda_{n},-I_{K}\right] \mu_{1}^{(n)} \\
& =\Pi_{n}^{\top}\left[\Sigma_{1}^{(n)-1}-\left[\begin{array}{cc}
\Sigma_{1}^{(n-1)-1} & 0 \\
0 & 0
\end{array}\right]\right] \mu_{1}^{(n)} \\
& =\Pi_{n}^{\top} \Sigma_{1}^{(n)-1} \mu_{1}^{(n)}-\Pi_{n-1}^{\top} \Sigma_{1}^{(n-1)-1} \mu_{1}^{(n-1)}
\end{aligned}
$$

Note that trivially $\Pi_{1}^{\top} \Sigma_{1}^{(1)-1} \mu_{1}^{(1)}=\Sigma_{11}^{-1} \mu_{1}$. Substituting (73), we see that (72) is a telescope sum such that writing $\Sigma_{1}^{(j)}=\Sigma$ and $\mu_{1}^{(j)}=\mu$, we get

$$
\theta_{1}=\Theta_{1} \Pi_{j}^{\top} \Sigma^{-1} \mu \text {. }
$$

Inserting (71) and (74) into (67) gives

$$
x_{0}^{\star}=\frac{1}{a R^{j-1}} \Pi_{j}^{\top} \Sigma^{-1}\left[\mu-\Pi_{j} R p\right] .
$$

This proves Theorem 1 .

\section{A.2 Technical appendix}

In this section we establish the existence of the value functions in Proposition 3 below and collect the required technical results for Gaussian functions. 


\section{Lemma 2}

Given the definition of a Gaussian function (56), the following properties hold:

(1) The product of $m$ Gaussian functions with parameters $\left(c^{(h)}, \vartheta^{(h)}, \Omega^{(h)}\right) \in \mathbb{R}_{++} \times$ $\mathbb{R}^{K} \times \mathcal{M}_{K}, h=1, \ldots, m$ is again a Gaussian function, that is,

$$
\prod_{h=1}^{m} g\left(x ; c^{(h)}, \vartheta^{(h)}, \Omega^{(h)}\right)=g(x ; c, \vartheta, \Omega),
$$

where the parameters $(c, \vartheta, \Omega) \in \mathbb{R}_{++} \times \mathbb{R}^{K} \times \mathcal{M}_{K}$ in (75) are given by

$$
\begin{aligned}
& \Omega=\left[\Omega^{(1)-1}+\ldots+\Omega^{(m)-1}\right]^{-1}, \quad \vartheta=\Omega\left[\Omega^{(1)-1} \vartheta^{(1)}+\ldots+\Omega^{(m)-1} \vartheta^{(m)}\right], \\
& \text { and } c=\frac{\prod_{h=1}^{m} g\left(0 ; c^{(h)}, \vartheta^{(h)}, \Omega^{(h)}\right)}{g(0 ; \vartheta, \Omega)} .
\end{aligned}
$$

(2) The integral of a Gaussian function $g(\cdot ; c, \vartheta, \Omega)$ over $\mathbb{R}^{K}$ satisfies

$$
\int_{\mathbb{R}^{K}} g(x ; c, \vartheta, \Omega) d x=\frac{c}{c(\Omega)}>0 .
$$

(3) For arbitrary $\kappa \in \mathbb{R}^{K}$ and invertible matrices $A \in \mathbb{R}^{K \times K}$, the following holds true:

$(E 1) \quad g(x ; c, \vartheta+\kappa, \Omega)=g(x-\kappa ; c, \vartheta, \Omega)$,

$(E 2) \quad g(x ; c, \vartheta, \Omega)=g(-x ; c,-\vartheta, \Omega)$,

(E3) $g(A x ; c, \vartheta, \Omega)=g\left(x ; c, A^{-1} \vartheta, A^{-1} \Omega A^{-\top}\right)$.

Proof. (1) follows by induction and straightforward calculations, (2) follows from the relation (57) and the properties of multivariate density functions, (3) can be verified from the definition given in (56) and direct calculations.

\section{Lemma 3}

Let $(\theta, \Theta) \in \mathbb{R}^{K} \times \mathcal{M}_{K}, c>0$ and $\alpha>0$ be given and the functions $u(\cdot ; \alpha)$ and $g(\cdot ; c, \theta, \Theta)$ be as defined in (3) and (56). Then

$$
\int_{\mathbb{R}^{K}} u\left(R y+x^{\top} p ; \alpha\right) g(p ; c, \theta, \Theta) d p=\frac{c}{c(\Theta)} u\left(R y+x^{\top} \theta-\frac{\alpha}{2} x^{\top} \Theta x ; \alpha\right) .
$$

Proof of Lemma 3. The integral kernel computes

$$
u\left(R y+x^{\top} p ; \alpha\right) g(p ; c, \theta, \Theta)=-c \exp \left\{-\alpha\left(R y+x^{\top} p\right)-\frac{1}{2}(p-\theta)^{\top} \Theta^{-1}(p-\theta)\right\}
$$

and using symmetry of the matrix $\Theta^{-1}=\Theta^{-\top}$, the exponent can be rewritten as

$$
\begin{aligned}
& -\alpha\left(R y+x^{\top} p\right)-\frac{1}{2}(p-\theta)^{\top} \Theta^{-1}(p-\theta) \\
= & -\alpha R y-\frac{1}{2}\left[(p-(\theta-\alpha \Theta x))^{\top} \Theta^{-1}(p-(\theta-\alpha \Theta x))+2 \alpha x^{\top} \theta-\alpha^{2} x^{\top} \Theta x\right] \\
= & -\alpha\left(R y+x^{\top} \theta-\frac{\alpha}{2} x^{\top} \Theta x\right)-\frac{1}{2}\left[(p-(\theta-\alpha \Theta x))^{\top} \Theta^{-1}(p-(\theta-\alpha \Theta x))\right] .
\end{aligned}
$$


Rearranging, the integrand becomes

$$
u\left(R y+x^{\top} p ; \alpha\right) g(p ; c, \theta, \Theta)=u\left(R y+x^{\top} \theta-\frac{\alpha}{2} x^{\top} \Theta x ; \alpha\right) g(p ; c, \theta-\alpha \Theta x, \Theta) .
$$

Since the first factor is independent of the integration variable $p$, the assertion follows from Lemma $2(2)$.

\section{Lemma 4}

Let the parameters $(\theta, \Theta) \in \mathbb{R}^{K} \times \mathcal{M}_{K}, c>0, \alpha>0$, prices $p \in \mathbb{R}^{K}$, and wealth $w \in \mathbb{R}$ be given. Then the following holds true:

(1) The optimization problem

$$
\max _{x, y}\left\{c u\left(R y+\theta^{\top} x-\frac{\alpha}{2} x^{\top} \Theta x ; \alpha\right) \mid y+p^{\top} x=w\right\}
$$

has a unique solution $\left(x^{\star}, y^{\star}\right) \in \mathbb{R}^{K} \times \mathbb{R}$ of the form

$$
x^{\star}=\frac{1}{\alpha} \Theta^{-1}(\theta-R p), \quad y^{\star}=w-p^{\top} x^{\star} .
$$

(2) The maximum value is given by

$$
u^{\star}:=c u\left(R y^{\star}+x^{\star \top} \theta-\frac{\alpha}{2} x^{\star \top} \Theta x^{\star} ; \alpha\right)=u(w ; \alpha R) g(R p ; c, \theta, \Theta) .
$$

Proof of Lemma 4. (1) Due to the strict monotonicity of the function $c u(\cdot ; \alpha)$ for each fixed $c>0$ and $\alpha>0$ maximizing the function

$$
\text { c } u\left(R y+x^{\top} \theta-\frac{\alpha}{2} x^{\top} \Theta x ; \alpha\right)=-c \exp \left\{-\alpha\left(R y+x^{\top} \theta-\frac{\alpha}{2} x^{\top} \Theta x\right)\right\} .
$$

is equivalent to maximizing the function $(x, y) \longmapsto R y+x^{\top} \theta-\frac{\alpha}{2} x^{\top} \Theta x$. From the Lagrangian function

$$
\mathcal{L}(x, y ; \lambda):=R y+x^{\top} \theta-\frac{\alpha}{2} x^{\top} \Theta x+\lambda\left(w-x^{\top} p-y\right)
$$

one obtains the first order conditions

$$
D_{x} \mathcal{L}\left(x^{\star}, y^{\star} ; \lambda\right)=\theta-\alpha \Theta x^{\star}-\lambda p \stackrel{!}{=} 0 \quad \text { and } \quad D_{y} \mathcal{L}\left(x^{\star}, y^{\star} ; \lambda\right)=R-\lambda \stackrel{!}{=} 0 .
$$

Combining this with the constraint $y+x^{\top} p=w$ yields the solution (76).

(2) Substituting the solution (76) into the objective function in (77) and exploiting the symmetry of the matrix $\Theta$ the maximum $u^{\star}$ reads:

$$
\begin{aligned}
u^{\star} & =c u\left(R w+x^{\star \top}(\theta-R p)-\frac{\alpha}{2} x^{\star \top} \Theta x^{\star} ; \alpha\right) \\
& =c u\left(R w+\frac{1}{2 \alpha}(\theta-R p)^{\top} \Theta^{-1}(\theta-R p) ; \alpha\right) \\
& =u(w ; \alpha R) g(R p ; c, \theta, \Theta)
\end{aligned}
$$

which proves the second assertion.

Equipped with this these prerequisites the following Proposition 3 establishes the desired representation of the value functions. 


\section{Proposition 3}

Let Assumption 4 be satisfied. Then for each $s=1, \cdots, j-1$ the value functions $V_{s}$ defined in (62) are well defined and take the form

$$
V_{s}\left(w_{s}, p_{1}^{s}\right)=u\left(w_{s} ; a R^{j-s}\right) g\left(p_{s} ; c_{s}, \vartheta_{s}, \Omega_{s}\right),
$$

with parameters ${ }^{7}$

$$
\begin{aligned}
\Omega_{s} & :=\left(\sum_{n=s+1}^{j} \Gamma_{n}^{(n-s) \top} \Sigma_{n}^{-1} \Gamma_{n}^{(n-s)}\right)^{-1} \\
\vartheta_{s} & :=\Omega_{s} \sum_{n=s+1}^{j} \Gamma_{n}^{(n-s) \top} \Sigma_{n}^{-1} \mu_{n \mid s-1} \\
c_{s} & :=\frac{\prod_{n=s+1}^{j} g\left(0 ; \gamma_{n}, \mu_{n \mid s-1}, \Sigma_{n}\right)}{g\left(0 ; \vartheta_{s}, \Omega_{s}\right)}
\end{aligned}
$$

where $\gamma_{j}:=1$ and

$$
\gamma_{n}:=\left(\frac{\operatorname{det} \Sigma_{n}^{-1}}{\operatorname{det}\left(\Sigma_{n}^{-1}+\Omega_{n}^{-1}\right)}\right)^{\frac{1}{2}}, \quad n=1, \ldots, j-1 .
$$

Proof of Proposition 3. The proof consists of two steps. In the first one, we verify the claim directly for $s=j-1$. The second step uses an induction argument

Step 1. Let $s=j-1$ and let wealth $w_{j-1}$ and realizations of prices $p_{1}, \ldots, p_{j-1}$ be given. By (62) the value function $V_{j-1}$ is

$$
V_{j-1}\left(w_{j-1}, p_{1}^{j-1}\right)=\max _{x, y}\left\{\int_{\mathbb{R}^{K}} u\left(R y+p^{\top} x ; a\right) f\left(p ; \mu_{j \mid j-1}, \Sigma_{j}\right) \mid y+x^{\top} p_{j-1}=w_{j-1}\right\} .
$$

Using (57) and applying Lemma 3 and Lemma $4, V_{j-1}$ becomes

$$
V_{j-1}\left(w_{j-1}, p_{1}^{j-1}\right)=u\left(w_{j-1} ; a R\right) g\left(R p_{j-1} ; \mu_{j \mid j-1}, \Sigma_{j}\right)
$$

Note that $R p_{j-1}-\mu_{j \mid j-1}=\Gamma_{j}^{(1)} p_{j-1}-\mu_{j \mid j-2}$ and therefore, exploiting properties (E1) and (E3) in Lemma 2,

$$
g\left(R p_{j-1} ; \mu_{j \mid j-1}, \Sigma_{j}\right)=g\left(\Gamma_{j}^{(1)} p_{j-1} ; \mu_{j \mid j-2}, \Sigma_{j}\right)=g\left(p_{j-1}, c_{j-1}, \vartheta_{j-1}, \Omega_{j-1}\right)
$$

where $c_{j-1}=1, \Omega_{j-1}=\Gamma_{j}^{(1) \top} \Sigma_{j}^{-1} \Gamma_{j}^{(1)}$ and $\vartheta_{j-1}=\Gamma_{j}^{(1)-1} \mu_{j \mid j-2}$. This proves the assertion for $s=j-1$. In particular, we have verified Proposition 3 for the case $j=2$.

Step 2. Let $s \in\{1, \ldots, j-2\}$ and assume that the claim holds for $s+1 \leq j-1$. We show by induction that the claim is true also for $s$. Let $w_{s}$ and $p_{1}, \ldots, p_{s}$ be given. Under the induction hypothesis the value function $V_{s}$ defined by (62) takes the form

$$
\begin{aligned}
& V_{s}\left(w_{s}, p_{1}^{s}\right) \\
= & \max _{y+p_{s}^{\top} x=w_{s}}\left\{\int_{\mathbb{R}^{K}} u\left(R y+p^{\top} x ; \frac{a}{R^{s+1-j}}\right) g\left(p ; c_{s+1}, \vartheta_{s+1}, \Omega_{s+1}\right) f\left(p ; \mu_{s+1 \mid s}, \Sigma_{s+1}\right) d p\right\}
\end{aligned}
$$

7 Observe that $\Omega_{s}$ is symmetric and positive definite, since the first term appearing in the sum is symmetric and positive definite due to (58) and Assumption 4 while the remaining terms are all symmetric and positive semi-definite. Moreover, $c_{j-1}=1$. 
Using (57) we obtain from Lemma 2(1)

$$
g\left(p ; c_{s+1}, \vartheta_{s+1}, \Omega_{s+1}\right) f\left(p ; \mu_{s+1 \mid s}, \Sigma_{s+1}\right)=g\left(p ; \hat{c}_{s+1}, \theta_{s+1}, \Theta_{s+1}\right)
$$

where

$$
\begin{aligned}
\Theta_{s+1} & :=\left[\Sigma_{s+1}^{-1}+\Omega_{s+1}^{-1}\right]^{-1} \\
\theta_{s+1} & :=\Theta_{s+1}\left[\Sigma_{s+1}^{-1} \mu_{s+1 \mid s}+\Omega_{s+1}^{-1} \vartheta_{s+1}\right] \\
\hat{c}_{s+1} & :=\frac{g\left(0 ; c_{s+1}, \vartheta_{s+1}, \Omega_{s+1}\right) g\left(0 ; c\left(\Sigma_{s+1}\right), \mu_{s+1 \mid s}, \Sigma_{s+1}\right)}{g\left(0 ; \theta_{s+1}, \Theta_{s+1}\right)} .
\end{aligned}
$$

Inserting (81) into (80) and applying Lemma 3 and 4 yields

$$
V_{s}\left(w_{s}, p_{1}^{s}\right)=u\left(w_{s} ; a R^{j-s}\right) g\left(R p_{s} ; \frac{\hat{c}_{s+1}}{c\left(\Theta_{s+1}\right)}, \theta_{s+1}, \Theta_{s+1}\right)
$$

The assertion will follow, if we can show that

$$
g\left(R p_{s} ; \frac{\hat{c}_{s+1}}{c\left(\Theta_{s+1}\right)}, \theta_{s+1}, \Theta_{s+1}\right)=g\left(p_{s} ; c_{s}, \vartheta_{s}, \Omega_{s}\right) .
$$

Noting from the definition (79) that $g\left(0 ; c_{s+1}, \vartheta_{s+1}, \Omega_{s+1}\right)=\prod_{n=s+2}^{j} g\left(0 ; \gamma_{n}, \mu_{n \mid s}, \Sigma_{n}\right)$ and $\frac{c\left(\Sigma_{s+1}\right)}{c\left(\Theta_{s+1}\right)}=\left(\frac{\operatorname{det} \Sigma_{s+1}^{-1}}{\operatorname{det}\left(\Sigma_{s+1}^{-1}+\Omega_{s+1}^{-1}\right)}\right)^{\frac{1}{2}}=\gamma_{s+1}$ we expand the l.h.s. of (83) using (81) to get

$$
\begin{aligned}
& g\left(R p_{s} ; \frac{\hat{c}_{s+1}}{c\left(\Theta_{s+1}\right)}, \theta_{s+1}, \Theta_{s+1}\right) \\
&= g\left(R p_{s} ; c_{s+1}, \vartheta_{s+1}, \Omega_{s+1}\right) g\left(R p_{s} ; \gamma_{s+1}, \mu_{s+1 \mid s}, \Sigma_{s+1}\right) \\
&= g\left(0 ; c_{s+1}, \vartheta_{s+1}, \Omega_{s+1}\right) \cdot \exp \left\{-\frac{1}{2} R p_{s}^{\top} \Omega_{s+1}^{-1} R p_{s}+R p_{s}^{\top} \Omega_{s+1}^{-1} \vartheta_{s+1}\right\} \\
& \cdot g\left(0 ; \gamma_{s+1}, \mu_{s+1 \mid s}, \Sigma_{s+1}\right) \cdot \exp \left\{-\frac{1}{2} R p_{s}^{\top} \Sigma_{s+1}^{-1} R p_{s}+R p_{s}^{\top} \Sigma_{s+1}^{-1} \mu_{s+1 \mid s}\right\} \\
&=\left(\prod_{n=s+1}^{j} g\left(0 ; \gamma_{n}, \mu_{n \mid s}, \Sigma_{n}\right)\right) \cdot \exp \left\{-\frac{1}{2} R p_{s}^{\top}\left(\Sigma_{s+1}^{-1}+\Omega_{s+1}^{-1}\right) R p_{s}\right\} \\
& \cdot \exp \left\{R p_{s}^{\top}\left(\Sigma_{s+1}^{-1} \mu_{s+1 \mid s}+\Omega_{s+1}^{-1} \vartheta_{s+1}\right)\right\} .
\end{aligned}
$$

All three terms appearing on the r.h.s. of (84) will now be rewritten separately.

1. Term. For each $n=s+1, \ldots, j$, (60) reads $\mu_{n \mid s}=\Lambda_{n}^{(n-s)} p_{s}+\mu_{n \mid s-1}$ as above. Using property $(E 1)$ of Gaussian functions

$$
\begin{aligned}
& g\left(0 ; \gamma_{n}, \mu_{n \mid s}, \Sigma_{n}\right)=g\left(-\Lambda_{n}^{(n-s)} p_{s}, \gamma_{n}, \mu_{n \mid s-1}, \Sigma_{n}\right) \\
& =g\left(0, \gamma_{n}, \mu_{n \mid s-1}, \Sigma_{n}\right) \cdot \exp \left\{-\frac{1}{2} p_{s}^{\top} \Lambda_{n}^{(n-s) \top} \Sigma_{n}^{-1}\left(\Lambda_{n}^{(n-s)} p_{s}+2 \mu_{n \mid s-1}\right)\right\} .
\end{aligned}
$$

By (79) we have $\prod_{n=s+1}^{j} g\left(0 ; \gamma_{n}, \mu_{n \mid s-1}, \Sigma_{n}\right)=g\left(0 ; c_{s}, \vartheta_{s}, \Omega_{s}\right)$, such that the first term on the r.h.s. of (84) takes the form

$$
\begin{aligned}
\prod_{n=s+1}^{j} g\left(0 ; \gamma_{n}, \mu_{n \mid s}, \Sigma_{n}\right)= & g\left(0 ; c_{s}, \vartheta_{s}, \Omega_{s}\right) \\
& \cdot \prod_{n=s+1}^{j} \exp \left\{-\frac{1}{2} p_{s}^{\top} \Lambda_{n}^{(n-s) \top} \Sigma_{n}^{-1}\left(\Lambda_{n}^{(n-s)} p_{s}+2 \mu_{n \mid s-1}\right)\right\} .
\end{aligned}
$$


2. Term. The definition (79) of $\Omega_{s+1}$ and (61) imply that

$$
\Sigma_{s+1}^{-1}+\Omega_{s+1}^{-1}=\sum_{n=s+1}^{j} \Gamma_{n}^{(n-s-1) \top} \Sigma_{n}^{-1} \Gamma_{n}^{(n-s-1)},
$$

such that the second term on the r.h.s. of (84) takes the form

$$
\begin{aligned}
& \exp \left\{-\frac{1}{2} R p_{s}^{\top}\left(\Sigma_{s+1}^{-1}+\Omega_{s+1}^{-1}\right) R p_{s}\right\} \\
= & \prod_{n=s+1}^{j} \exp \left\{-\frac{1}{2} R p_{s}^{\top}\left(\Gamma_{n}^{(n-s-1) \top} \Sigma_{n}^{-1} \Gamma_{n}^{(n-s-1)}\right) R p_{s}\right\} .
\end{aligned}
$$

3. Term. We have from the definition (79) of $\Omega_{s+1}$ and $\vartheta_{s+1}$

$$
\Sigma_{s+1}^{-1} \mu_{s+1 \mid s}+\Omega_{s+1}^{-1} \vartheta_{s+1}=\sum_{n=s+1}^{j} \Gamma_{n}^{(n-s-1) \top} \Sigma_{n}^{-1}\left(\Lambda_{n}^{(n-s)} p_{s}+\mu_{n \mid s-1}\right),
$$

such that the third term on the r.h.s. of (84) takes the form

$$
\begin{aligned}
& \exp \left\{R p_{s}^{\top}\left(\Sigma_{s+1}^{-1} \mu_{s+1 \mid s}+\Omega_{s+1}^{-1} \vartheta_{s+1}\right)\right\} \\
= & \prod_{n=s+1}^{j} \exp \left\{R p_{s}^{\top} \Gamma_{n}^{(n-s-1) \top} \Sigma_{n}^{-1}\left(\Lambda_{n}^{(n-s)} p_{s}+\mu_{n \mid s-1}\right)\right\} .
\end{aligned}
$$

Inserting (85), (86), and (87) and utilizing that (61) implies $\Gamma_{n}^{(n-s)}=R \Gamma_{n}^{(n-s-1)}-\Lambda_{n}^{(n-s)}$ for each $n=s+1, \ldots, j$, we can rewrite (84) as

$$
\begin{aligned}
& g\left(R p_{s} ; \frac{\hat{c}_{s+1}}{c\left(\Theta_{s+1}\right)}, \theta_{s+1}, \Theta_{s+1}\right) \\
= & \left.g\left(0 ; c_{s}, \vartheta_{s}, \Omega_{s}\right) \prod_{n=s+1}^{j} \exp \left\{p_{s}^{\top}\left[R \Gamma_{n}^{(n-s-1)}-\Lambda_{n}^{(n-s)}\right]^{\top} \Sigma_{n}^{-1} \mu_{n \mid s-1}\right)\right\} \\
& \cdot \prod_{n=s+1}^{j} \exp \left\{-\frac{1}{2} p_{s}^{\top}\left[R \Gamma_{n}^{(n-s-1)}-\Lambda_{n}^{(n-s)}\right]^{\top} \Sigma_{n}^{-1}\left[R \Gamma_{n}^{(n-s-1)}-\Lambda_{n}^{(n-s)}\right] p_{s}\right\} \\
= & g\left(0 ; c_{s}, \vartheta_{s}, \Omega_{s}\right) \prod_{n=s+1}^{j} \exp \left\{p_{s}^{\top} \Gamma_{n}^{(n-s) \top} \Sigma_{n}^{-1} \mu_{n \mid s-1}\right\} \prod_{n=s+1}^{j} \exp \left\{-\frac{1}{2} p_{s}^{\top} \Gamma_{n}^{(n-s) \top} \Sigma_{n}^{-1} \Gamma_{n}^{(n-s)} p_{s}\right\} \\
= & g\left(0 ; c_{s}, \vartheta_{s}, \Omega_{s}\right) \exp \left\{p_{s}^{\top} \sum_{n=s+1}^{j} \Gamma_{n}^{(n-s) \top} \Sigma_{n}^{-1} \mu_{n \mid s-1}\right\} \exp \left\{-\frac{1}{2} p_{s}^{\top} \sum_{n=s+1}^{j} \Gamma_{n}^{(n-s) \top} \Sigma_{n}^{-1} \Gamma_{n}^{(n-s)} p_{s}\right\} \\
= & g\left(0 ; c_{s}, \vartheta_{s}, \Omega_{s}\right) \exp \left\{p_{s}^{\top} \Omega_{s}^{-1} \vartheta_{s}\right\} \exp \left\{-\frac{1}{2} p_{s}^{\top} \Omega_{s}^{-1} p_{s}\right\} \\
= & g\left(p_{s} ; c_{s}, \vartheta_{s}, \Omega_{s}\right) .
\end{aligned}
$$

This proves (83) and completes the proof of Proposition 3. 


\section{References}

Anderson, T. W. (1984): An Introduction to Multivariate Statistical Analysis. John Wiley \& Sons, New York a.o.

Arnold, L. (1998): Random Dynamical Systems. Springer-Verlag, Berlin a.o.

Böhm, V. \& C. Chiarella (2000): "Mean Variance Preferences, Expectations Formations, and the Dynamics of Random Asset Prices", Discussion Paper No. 448, University of Bielefeld, forthcoming in Mathematical Finance.

Böhm, V., N. Deutscher \& J. Wenzelburger (2000): "Endogenous Random Asset Prices in Overlapping Generations Economies", Mathematical Finance, 10(1), 23-38.

Böhm, V. \& J. Wenzelburger (2002): "On the Performance of Efficient Portfolios", Discussion Paper 493, Department of Economics, University of Bielefeld.

Brock, W. A. \& C. H. Hommes (1997): "Models of Complexity in Economics and Finance", in Systems Dynamics in Economic and Finance Models, ed. by B. Hanzon, C. Heij, J. Schumacher \& C. Praagman, chap. 1, S. 3-44. Wiley, New York.

(1998): "Heterogeneous Beliefs and Routes to Chaos in a Simple Asset Pricing Model.", Journal of Economic Dynamics and Control, 22, 1235-1274.

Chen, A. H., F. C. Jen \& S. Zionts (1971): "The Optimal Portfolio Revision Policy", The Journal of Business, 44.

Chiarella, C. \& X.-Z. He (2002): "The Dynamics of Beliefs and Learning under $a_{L^{-}}$ Processes; the Heterogeneous Case", Journal of Economic Dynamics and Control, to appear.

De Long, J. B., A. Shleifer, L. H. Summers \& R. J. Waldmann (1990): "Noise Traders Risk in Financial Markets", Journal of Political Economy, 98, 703-738.

Hakansson, N. H. (1970): "Optimal Investment and Consumption Strategies under Risk for a Class of Utility Functions", Econometrica, 38.

(1983): "Multi-Period Mean-Variance Analysis: Toward a General Theory of Portfolio Choice", The Journal of Finance, 38.

Hillebrand, M. (2003): "Sequentielle Portefeuille-Entscheidungen mit mehrperiodigem Planungszeitraum - Ein Finanzmarktmodell mit endogener Preisbildung", Diplomarbeit, Fakultät für Wirtschaftswissenschaften, Universität Bielefeld.

Ingersoll, J. E. (1987): Theory of Financial Decision Making. Rowman \& Littlefield, Totowa (NJ).

Lintner, J. (1965): "The Valuation of Risky Assets and Selection of Risky Investment in Stock Portfolios and Capital Budgets", Review of Economics and Statistics, 47, 13-37. 
Markowitz, H. (1952): "Portfolio Selection", Journal of Finance, 7, 77-91.

Mossin, J. (1966): "Equilibrium in a Capital Asset Market", Econometrica, 34, 268290.

Ouellette, D. V. (1981): "Schur Complements and Statistics", Linear Algebra and its Applications, 36, 187-295.

PliskA, S. (1997): Introduction to Mathematical Finance. Blackwell Publishers Inc, Massachusetts.

Sharpe, W. F. (1964): "Capital Asset Pricing: A Theory of Market Equilibrium Under Conditions of Risk", Journal of Finance, 19, 425-442.

Stapleton, R. \& M. Subrahmanyam (1978): "A Multiperiod Equilibrium Asset Pricing Model", Econometrica, 46, 1077-1096.

Tobin, J. (1958): "Liquidity Preference as Behavior Towards Risk", Review of Economic Studies, 25, 65-86.

Tong, Y. (1990): The Multivariate Normal Distribution. Springer Verlag, New York a.o.

Wenzelburger, J. (2001): "Learning in Linear Models with Expectational Leads", Discussion Paper No. 478, University of Bielefeld.

(2004): "Learning to Predict Rationally when Beliefs are Heterogeneous", Journal of Economic Dynamics and Control, 10(28), 2075-2104. 